\title{
Stability and convergence of efficient Navier-Stokes solvers via a commutator estimate ${ }^{0}$
}

\author{
Jian-Guo Liu ${ }^{1}$, Jie Liu², and Robert L. Pego ${ }^{3}$
}

October 2005 (revised February 2006)

\begin{abstract}
For strong solutions of the incompressible Navier-Stokes equations in bounded domains with velocity specified at the boundary, we establish the unconditional stability and convergence of discretization schemes that decouple the updates of pressure and velocity through explicit time-stepping for pressure. These schemes require no solution of stationary Stokes systems, nor any compatibility between velocity and pressure spaces to ensure an inf-sup condition, and are representative of a class of highly efficient computational methods that have recently emerged. The proofs are simple, based upon a new, sharp estimate for the commutator of the Laplacian and Helmholtz projection operators. This allows us to treat an unconstrained formulation of the Navier-Stokes equations as a perturbed diffusion equation.
\end{abstract}

\section{Introduction}

The Navier-Stokes equations for incompressible fluid flow in a domain $\Omega$ in $\mathbb{R}^{N}$ $(N \geq 2)$ with specified velocity on the boundary $\Gamma$ take the form

$$
\begin{aligned}
\partial_{t} \mathbf{u}+\mathbf{u} \cdot \nabla \mathbf{u}+\nabla p=\nu \Delta \mathbf{u}+\mathbf{f} & & \text { in } \Omega, \\
\nabla \cdot \mathbf{u}=0 & & \text { in } \Omega, \\
\mathbf{u}=\mathbf{g} & & \text { on } \Gamma .
\end{aligned}
$$

Here $\mathbf{u}$ is the fluid velocity, $p$ is the pressure, and $\nu$ is the kinematic viscosity coefficient, which we assume to be a fixed positive constant. We assume $\Omega$ is bounded and connected and $\Gamma=\partial \Omega$ is $C^{3}$.

\footnotetext{
${ }^{0}$ This is a preprint of an article accepted for publication in Communications on Pure and Applied Mathematics, (c)2007 Wiley Periodicals Inc.

${ }^{1}$ Department of Mathematics \& Institute for Physical Science and Technology, University of Maryland, College Park MD 20742. Email: jliu@math.umd.edu

${ }^{2}$ Department of Mathematics, University of Maryland, College Park MD 20742. Email: jieliu@math.umd.edu

${ }^{3}$ Department of Mathematical Sciences, Carnegie Mellon University, Pittsburgh, PA 15213. Email: rpego@cmu.edu
} 
The pressure has long been a main source of trouble for understanding and computing solutions to these equations. Pressure plays a role like a Lagrange multiplier to enforce the incompressibility constraint. This leads to computational difficulties, typically related to the lack of an evolution equation for updating the pressure dynamically, and a lack of useful boundary conditions for determining the pressure by solving boundary-value problems.

These issues are particularly important in domains with boundary. Much of the scientific and technological impact of the Navier-Stokes equations derives from the effect of no-slip boundary conditions in creating physical phenomena such as lift, drag, boundary-layer separation and vortex shedding, for which the behavior of the pressure near boundaries is of great significance. Consequently, computing with high accuracy and stability near boundaries is a major goal.

Yet most existing computational methods able to handle the difficulties are sophisticated and lack the robustness and flexibility that would be useful to address more complex problems. For example, many finite element methods require the solution of Stokes equations that couple velocity and pressure, and great care is required to arrange that the approximation spaces for these variables are compatible in the sense that an inf-sup condition holds that ensures stability [GR, FB]. Stabilized finite element methods have been developed that allow one to circumvent such compatibility conditions, but at the cost of additional complexity [BBGS]. Projection methods, pioneered by Chorin [Ch1, Ch2] and Temam [Te2], achieved a separation between updates of velocity and pressure through a splitting strategy. It has proved difficult, however, to achieve high-order accuracy for pressure near boundaries [OID] — uniform second-order accuracy was attained only a few years ago [Ti, BCM].

Recently, a number of workers have developed efficient and accurate numerical methods that separate velocity and pressure updates without generating numerical boundary layers, in ways that turn out to be closely related. The main objective of this paper is to develop, for a representative class of such schemes, a theory of stability and convergence to strong solutions, with the regularity

$$
\begin{aligned}
& \mathbf{u} \in L^{2}\left(0, T ; H^{2}\left(\Omega, \mathbb{R}^{N}\right)\right) \cap H^{1}\left(0, T ; L^{2}\left(\Omega, \mathbb{R}^{N}\right)\right), \\
& \nabla p \in L^{2}\left(0, T ; L^{2}\left(\Omega, \mathbb{R}^{N}\right)\right) .
\end{aligned}
$$

In particular we establish unconditional stability and convergence for a class of fully discrete finite element methods which need not satisfy any compatibility requirement related to an inf-sup condition, for the case of no-slip boundary conditions. Our theory is based on a new, sharp estimate in $L^{2}$-norm for the commutator of the Laplacian and Helmholtz projection operators. A side benefit of the theory is a particularly simple proof of local existence and uniqueness for strong solutions in bounded domains.

For simplicity, at first we consider no-slip boundary conditions, taking $\mathbf{g}=0$. Below, we let $\langle f, g\rangle_{\Omega}=\int_{\Omega} f g$ denote the $L^{2}$ inner product of functions $f$ and $g$ in $\Omega$, and let $\|\cdot\|_{\Omega}$ denote the corresponding norm in $L^{2}(\Omega)$. We drop the subscript on the inner product and norm when the domain of integration is 
understood in context.

The schemes that we study are based on the following time-discretization scheme, implicit only in the viscosity term and explicit in the pressure and nonlinear terms. Given an approximation $\mathbf{u}^{n}$ to the velocity at time $n \Delta t$, determine $\nabla p^{n}$ from a weak-form pressure Poisson equation, requiring

$$
\left\langle\nabla p^{n}, \nabla \phi\right\rangle=\left\langle\mathbf{f}^{n}-\mathbf{u}^{n} \cdot \nabla \mathbf{u}^{n}+\nu \Delta \mathbf{u}^{n}-\nu \nabla \nabla \cdot \mathbf{u}^{n}, \nabla \phi\right\rangle \quad \forall \phi \in H^{1}(\Omega),
$$

then determine $\mathbf{u}^{n+1}$ from the elliptic boundary-value problem

$$
\begin{array}{r}
\frac{\mathbf{u}^{n+1}-\mathbf{u}^{n}}{\Delta t}-\nu \Delta \mathbf{u}^{n+1}=\mathbf{f}^{n}-\mathbf{u}^{n} \cdot \nabla \mathbf{u}^{n}-\nabla p^{n}, \\
\left.\mathbf{u}^{n+1}\right|_{\Gamma}=0 .
\end{array}
$$

Ultimately we will consider corresponding fully discrete finite-element methods that use $C^{1}$ elements for the velocity field and $C^{0}$ elements for the pressure.

The discretization scheme above is a prototype for a class of highly efficient numerical methods for incompressible flow obtained using different kinds of spatial discretization and higher-order time differencing [Ti, HP, Pe, GuS, JL]. The computation of velocity updates is completely divorced from the computation of pressure from the kinematic pressure Poisson equation. No stationary Stokes solver is necessary to handle implicitly differenced pressure terms. The computation of incompressible Navier-Stokes dynamics in a general domain is thereby reduced to solving a heat equation and a Poisson equation at each time step.

This decoupling of velocity and pressure updates is also characteristic of projection methods, which are closely related. But a distinguishing aspect of the new schemes is the consistent way the boundary condition is implemented, and their improved accuracy and flexibility. We refer to Brown et al. [BCM] for a study of second-order accuracy near the boundary for several modern improvements of the projection method. We will discuss the connections between the scheme (4)-(6) above, recent improvements of the projection method, and the gauge method [EL], in some detail in section 2 .

The presence of the grad-div term in the pressure Poisson equation (4) is a key feature that allows us to prove the stability of the schemes we consider. To begin to explain the usefulness of this term, we next describe an unconstrained formulation of the Navier-Stokes equations that underlies the scheme (4)-(6).

Recall that a standard way to analyze the Navier-Stokes equations uses the Helmholtz-Hodge decomposition. We let $\mathcal{P}$ denote the Helmholtz projection operator onto divergence-free fields, defined as follows. Given any $\mathbf{a} \in L^{2}\left(\Omega, \mathbb{R}^{N}\right)$, there is a unique $q \in H^{1}(\Omega)$ with $\int_{\Omega} q=0$ such that $\mathcal{P} \mathbf{a}:=\mathbf{a}+\nabla q$ satisfies

$$
0=\langle\mathcal{P} \mathbf{a}, \nabla \phi\rangle=\langle\mathbf{a}+\nabla q, \nabla \phi\rangle \quad \forall \phi \in H^{1}(\Omega) .
$$

The pressure $p$ in (1) can be regarded as determined by taking $\mathbf{a}=\mathbf{u} \cdot \nabla \mathbf{u}-\mathbf{f}-$ $\nu \Delta \mathbf{u}$. Then (1) is rewritten as

$$
\partial_{t} \mathbf{u}+\mathcal{P}(\mathbf{u} \cdot \nabla \mathbf{u}-\mathbf{f}-\nu \Delta \mathbf{u})=0 .
$$


In this formulation, solutions formally satisfy $\partial_{t}(\nabla \cdot \mathbf{u})=0$. Consequently the zero-divergence condition (2) needs to be imposed only on initial data. Nevertheless, the pressure is determined from (8) in principle even for velocity fields that do not respect the incompressibility constraint. But then the dissipation in (8) appears degenerate due to the fact that $\mathcal{P}$ annihilates gradients. Thus, the analysis of (8) is usually restricted to spaces of divergence-free fields.

It is possible to determine the pressure differently when the velocity field has non-zero divergence. Instead of (8), we consider

$$
\partial_{t} \mathbf{u}+\mathcal{P}(\mathbf{u} \cdot \nabla \mathbf{u}-\mathbf{f}-\nu \Delta \mathbf{u})=\nu \nabla(\nabla \cdot \mathbf{u}) .
$$

Of course there is no difference as long as $\nabla \cdot \mathbf{u}=0$. But the incompressibility constraint is enforced in a more robust way, because the divergence of velocity satisfies a weak form of the diffusion equation with no-flux (Neumann) boundary conditions - Due to (7), for all appropriate test functions $\phi$ we have

$$
\left\langle\partial_{t} \mathbf{u}, \nabla \phi\right\rangle=\langle\nu \nabla(\nabla \cdot \mathbf{u}), \nabla \phi\rangle .
$$

Due to the fact that $\int_{\Omega} \nabla \cdot \mathbf{u}=0$, the divergence of velocity is smoothed and decays exponentially in $L^{2}$ norm. Naturally, if $\nabla \cdot \mathbf{u}=0$ initially, this remains true for all later time, and one has a solution of the standard Navier-Stokes equations (1)-(3).

The unconstrained formulation (9) can be recast in the form (1). Taking $\mathbf{a}=\mathbf{u} \cdot \nabla \mathbf{u}-\mathbf{f}-\nu \Delta \mathbf{u}+\nu \nabla \nabla \cdot \mathbf{u}$, and noting $\mathcal{P} \nabla \nabla \cdot \mathbf{u}=0$, we find the projection term in (9) has the form $\mathcal{P} \mathbf{a}=\mathbf{a}+\nabla p$ with the pressure determined as in (7). Thus, with no-slip boundary conditions, (9) is equivalent to

$$
\begin{aligned}
\partial_{t} \mathbf{u}+\mathbf{u} \cdot \nabla \mathbf{u}+\nabla p=\nu \Delta \mathbf{u}+\mathbf{f} & \text { in } \Omega, \\
\langle\nabla p, \nabla \phi\rangle=\langle\mathbf{f}-\mathbf{u} \cdot \nabla \mathbf{u}+\nu \Delta \mathbf{u}-\nu \nabla \nabla \cdot \mathbf{u}, \nabla \phi\rangle & \forall \phi \in H^{1}(\Omega), \\
\mathbf{u}=0 & \text { on } \Gamma .
\end{aligned}
$$

The scheme (4)-(6) comes directly from discretization of this system.

An alternative form of (9) proves illuminating. First, notice the following.

Lemma 1 Let $\Omega \subset \mathbb{R}^{N}$ be a bounded domain, and let $\mathbf{u}$ be a vector field in $L^{2}\left(\Omega, \mathbb{R}^{N}\right)$. Then in the sense of distributions, one has

$$
\nabla \nabla \cdot \mathbf{u}=\Delta(I-\mathcal{P}) \mathbf{u}
$$

Proof: We have $(I-\mathcal{P}) \mathbf{u}=\nabla q$ where $q \in H^{1}(\Omega)$ and $\Delta q=\nabla \cdot \mathbf{u}$. Then $\nabla \nabla \cdot \mathbf{u}=\nabla \Delta q=\Delta \nabla q$ and the result follows.

Using this result, we see that (9) takes the equivalent form

$$
\partial_{t} \mathbf{u}+\mathcal{P}(\mathbf{u} \cdot \nabla \mathbf{u}-\mathbf{f})+\nu[\Delta, \mathcal{P}] \mathbf{u}=\nu \Delta \mathbf{u},
$$

where $[\Delta, \mathcal{P}]=\Delta \mathcal{P}-\mathcal{P} \Delta$ is the commutator of the Laplacian and Helmholtz projection operators. One may expect this commutator to be "small" in some sense. Indeed, it vanishes in the case when the domain is a box with periodic boundary conditions. With no-slip boundary conditions, it turns out that the commutator is a second-order operator bounded by the following sharp estimate. This estimate is the key to our stability theory. 
Theorem 1 Let $\Omega \subset \mathbb{R}^{N}$ (N $\geq 2$ ) be a connected bounded domain with $C^{3}$ boundary. Then for any $\varepsilon>0$, there exists $C \geq 0$ such that for all vector fields $\mathbf{u} \in H^{2} \cap H_{0}^{1}\left(\Omega, \mathbb{R}^{N}\right)$,

$$
\int_{\Omega}|(\Delta \mathcal{P}-\mathcal{P} \Delta) \mathbf{u}|^{2} \leq\left(\frac{1}{2}+\varepsilon\right) \int_{\Omega}|\Delta \mathbf{u}|^{2}+C \int_{\Omega}|\nabla \mathbf{u}|^{2} .
$$

The coefficient $\frac{1}{2}+\varepsilon$ is essentially sharp - we show in Appendix A using Fourier analysis that when $\Omega=\mathbb{R}_{+}^{N}$ is the half-space where $x_{N}>0$, then

$$
\int_{\Omega}|(\Delta \mathcal{P}-\mathcal{P} \Delta) \mathbf{u}|^{2} \leq \frac{1}{2} \int_{\Omega}|\Delta \mathbf{u}|^{2}
$$

for all $\mathbf{u} \in H^{2} \cap H_{0}^{1}\left(\Omega, \mathbb{R}^{N}\right)$, and equality holds for some $\mathbf{u}$.

Notice that by Lemma $1,[\Delta, \mathcal{P}] \mathbf{u}$ is a gradient:

$$
(\Delta \mathcal{P}-\mathcal{P} \Delta) \mathbf{u}=(I-\mathcal{P}) \Delta \mathbf{u}-\nabla \nabla \cdot \mathbf{u}=(I-\mathcal{P})(\Delta-\nabla \nabla \cdot) \mathbf{u} .
$$

Thus the pressure in (11)-(12) is determined by

$$
\nabla p=(I-\mathcal{P})(\mathbf{f}-\mathbf{u} \cdot \nabla \mathbf{u})+\nu(\Delta \mathcal{P}-\mathcal{P} \Delta) \mathbf{u} .
$$

The first part of this pressure gradient is nonlinear but involves only derivatives up to first order. The second part is controlled by Theorem 1, in a way that significantly sharpens the standard bound $\|[\Delta, \mathcal{P}] \mathbf{u}\| \leq\|\Delta \mathbf{u}\|$ valid when $\nabla \cdot \mathbf{u}=$ 0 by $L^{2}$-orthogonality. Because we can take $\frac{1}{2}+\varepsilon<1$, for stability analysis the whole pressure gradient can be strictly controlled by the viscosity term, modulo lower-order terms. This explains why one can discretize the pressure explicitly. Thus, due to the estimate in Theorem 1, we can regard (14) or equivalently (9) or (11)-(12) as a controlled perturbation of the vector diffusion equation $\partial_{t} \mathbf{u}=\nu \Delta \mathbf{u}$, in contrast to the usual approach that regards the Navier-Stokes equations as a perturbation of the Stokes system $\partial_{t} \mathbf{u}+\nabla p=\nu \Delta \mathbf{u}, \nabla \cdot \mathbf{u}=0$.

We will prove Theorem 1 in section 3. Based on the estimate (15), we establish unconditional stability of the time-discretization scheme (4)-(6) in section 4. A straightforward compactness argument, given in section 5, yields convergence to the unique local strong solution to the unconstrained system (11)-(13) with initial values $\mathbf{u}(\cdot, 0)=\mathbf{u}_{\text {in }} \in H^{1}\left(\Omega, \mathbb{R}^{N}\right)$. When $\nabla \cdot \mathbf{u}_{\text {in }}=0$ this is the local strong solution of the standard Navier-Stokes equations (1)-(3).

The analysis of the time-discrete scheme easily adapts to proving unconditional stability and convergence for corresponding fully discrete finite-element methods with $C^{1}$ elements for velocity and $C^{0}$ elements for pressure as mentioned above. We carry out this analysis in section 6 . It is important to note that the finite-element spaces for velocity and pressure need not satisfy any compatibility requirement to enforce an inf-sup condition. The inf-sup condition (also known as the Ladyzhenskaya-Babuška-Brezzi condition in the context of the Stokes equation) has long been a central foundation for finite-element methods for all saddle-point problems. Its beautiful theory is a masterpiece documented 
in many finite-element books. In the usual approach, the inf-sup condition ensures the stability of the approximation, but this imposes a compatibility restriction on the finite-element spaces for velocity and pressure which is violated by many natural choices - piecewise polynomials of the same order, for example. Here, however, due to the fully dissipative nature of the unconstrained formulation (9), which follows as a consequence of Theorem 1, as far as our stability analysis in section 6 is concerned, the finite-element spaces for velocity and pressure can be completely unrelated.

We deal with nonhomogenous boundary conditions (g nonzero in (3)) in section 7 , and provide an estimate on divergence for the time-discrete scheme in section 8. We conclude with three appendices. In particular, in Appendix A we prove (16), and in Appendix B we study the range of the commutator $[\Delta, \mathcal{P}]$ acting on $H^{2}\left(\Omega, \mathbb{R}^{N}\right)$. This operator accounts for the contribution to the pressure gradient by the viscosity term in bounded domains. In three dimensions it turns out that its range is the space of square-integrable vector fields that are simultaneously gradients and curls (see Theorem 9).

\section{Related formulations and schemes}

\subsection{Stokes pressure and curl curl}

In order to discuss the connections between the schemes that we treat and related ones in the literature, we first need to make some points regarding the commutator $\Delta \mathcal{P}-\mathcal{P} \Delta$.

The commutator term in (18) supplies the part of the pressure due to the viscosity term. This is the entire pressure when one neglects nonlinear and forcing terms, as in the linear Stokes equation. Hence we call this part of the pressure the Stokes pressure associated with the vector field $\mathbf{u} \in H^{2}\left(\Omega, \mathbb{R}^{N}\right)$. The Stokes pressure $p_{\mathrm{S}}$ is defined from

$$
\nabla p_{\mathrm{S}}=(\Delta \mathcal{P}-\mathcal{P} \Delta) \mathbf{u}
$$

According to (17) and (7), $p_{\mathrm{S}}$ is determined as the mean-zero solution of

$$
\int_{\Omega} \nabla p_{\mathrm{S}} \cdot \nabla \phi=\int_{\Omega}(\Delta \mathbf{u}-\nabla \nabla \cdot \mathbf{u}) \cdot \nabla \phi \quad \forall \phi \in H^{1}(\Omega) .
$$

We observe that the Stokes pressure is a harmonic function determined by a meaningful boundary value problem. Formal integration by parts in (20) suggests that $p_{\mathrm{S}}$ is determined as the solution of the Neumann boundary-value problem

$$
\Delta p_{\mathrm{S}}=0 \quad \text { in } \Omega, \quad \mathbf{n} \cdot \nabla p_{\mathrm{S}}=\mathbf{n} \cdot(\Delta-\nabla \nabla \cdot) \mathbf{u} \quad \text { on } \Gamma .
$$

Indeed, this is true for any $\mathbf{u} \in H^{2}\left(\Omega, \mathbb{R}^{N}\right)$ : In the sense of distributions, the vector field $\mathcal{A} \mathbf{u}:=\Delta \mathbf{u}-\nabla \nabla \cdot \mathbf{u}$ has zero divergence, hence $\Delta p_{\mathrm{S}}=0$. Moreover, $\mathcal{A} \mathbf{u}$ and $\nabla p_{\mathrm{S}}$ belong to $L^{2}\left(\Omega, \mathbb{R}^{N}\right)$, so both lie in the space $H(\operatorname{div} ; \Omega)$ consisting 
of vector fields in $L^{2}\left(\Omega, \mathbb{R}^{N}\right)$ with divergence in $L^{2}(\Omega)$. By a well-known trace theorem (see [GR], theorem 2.5), the normal components of $\mathcal{A} \mathbf{u}$ and $\nabla p_{\mathrm{S}}$ belong to the Sobolev space $H^{-1 / 2}(\Gamma)$, and (20) implies

$$
0=\int_{\Gamma} \phi \mathbf{n} \cdot\left(\nabla p_{\mathrm{S}}-\Delta \mathbf{u}+\nabla \nabla \cdot \mathbf{u}\right), \quad \forall \phi \in H^{1}(\Omega) .
$$

From this we infer that the boundary condition in (21) holds in $H^{-1 / 2}(\Gamma)$.

In two and three dimensions, the Stokes pressure is generated by the tangential part of vorticity at the boundary. To see this, start with the identity

$$
\Delta \mathbf{u}-\nabla \nabla \cdot \mathbf{u}=-\nabla \times \nabla \times \mathbf{u},
$$

whence $\nabla p_{\mathrm{S}}=-(I-\mathcal{P})(\nabla \times \nabla \times \mathbf{u})$. Green's formula yields

$$
\int_{\Gamma} \mathbf{n} \cdot(\nabla \times \nabla \times \mathbf{u}) \phi=\int_{\Omega}(\nabla \times \nabla \times \mathbf{u}) \cdot \nabla \phi=-\int_{\Gamma}(\nabla \times \mathbf{u}) \cdot(\mathbf{n} \times \nabla \phi)
$$

and therefore from $(20), p_{\mathrm{S}}$ is also determined by the condition [JL]

$$
\int_{\Omega} \nabla p_{\mathrm{S}} \cdot \nabla \phi=\int_{\Gamma}(\nabla \times \mathbf{u}) \cdot(\mathbf{n} \times \nabla \phi) \quad \forall \phi \in H^{1}(\Omega) .
$$

Note that $\nabla \times \mathbf{u} \in H^{1 / 2}\left(\Gamma, \mathbb{R}^{N}\right)$, and $\mathbf{n} \times \nabla \phi \in H^{-1 / 2}\left(\Gamma, \mathbb{R}^{N}\right)$ by a standard trace theorem [GR, Theorem 2.11], since $\nabla \phi$ lies in $H(\operatorname{curl} ; \Omega)$, the space of vector fields in $L^{2}\left(\Omega, \mathbb{R}^{N}\right)$ with curl in $L^{2}$.

The unconstrained formulation of the Navier-Stokes equations in (11)-(12) now can be seen to be equivalent to a formulation analyzed by Grubb and Solonnikov in [GS1, GS2]. For Dirichlet-type boundary conditions, these authors obtain the contribution to pressure from viscosity by solving the boundary value problem (21). But they did not describe the connection between (21) and the commutator $\Delta \mathcal{P}-\mathcal{P} \Delta$. Grubb and Solonnikov performed an analysis of their formulation based on a general theory of parabolic pseudodifferential initialboundary value problems, and they argued that this formulation is parabolic in a nondegenerate sense. They also showed that for strong solutions, the divergence of velocity satisfies a diffusion equation with Neumann boundary conditions.

\subsection{Related schemes that damp divergence}

Recently a number of workers have independently developed splitting schemes that can be obtained directly from the time-discrete scheme in (4)-(6) through spatial discretization and including higher-order time differencing. In particular we mention works of Henshaw and Petersson [HP], Guermond and Shen [GuS], and Johnston and Liu [JL]. The common ingredient is that the pressure is determined from a Poisson equation using the curl-curl boundary condition as in (21). The first related use of this curl-curl boundary condition appears to be by Orszag et al. [OID], who used it as a way of enforcing consistency for a Neumann problem in the context of the projection method. 
In the introduction, we motivated the scheme (4)-(6) through adding a graddiv term that produces a diffusive damping of the divergence of velocity in the time-continuous formulation (9). In addition, or alternatively, one can add a term to produce linear damping of the divergence of velocity. Petersson employed this procedure in [Pe] (also see [HP]) as a way of further suppressing divergence errors. With constant divergence damping, his procedure is equivalent to adding $\lambda(I-\mathcal{P}) \mathbf{u}$ on the left-hand side of $(9)$.

Upon discretization, this produces several schemes close to ones that have figured significantly in the recent literature related to the projection method. In the first-order scheme as suggested by Petersson, the discretized momentum equation may be written in the form

$$
\frac{\mathbf{u}^{n+1}-\mathbf{u}^{n}}{\Delta t}+\lambda(I-\mathcal{P}) \mathbf{u}^{n}+\mathcal{P}\left(\mathbf{u}^{n} \cdot \nabla \mathbf{u}^{n}-\mathbf{f}^{n}\right)+\nu \nabla p_{\mathrm{s}}^{n}=\nu \Delta \mathbf{u}^{n+1},
$$

with $\nabla p_{\mathrm{S}}^{n}$ determined from $\mathbf{u}^{n}$ as in $(21)$; that is, $\nabla p_{\mathrm{S}}^{n}=(\Delta \mathcal{P}-\mathcal{P} \Delta) \mathbf{u}^{n}$ is the Stokes pressure determined by $\mathbf{u}^{n}$.

Taking $\lambda=1 / \Delta t$ in (26) and replacing $\mathbf{u}^{n}$ by $\mathcal{P} \mathbf{u}^{n}$ in the convection term results in the following method close to a first-order version of the projection method introduced by Timmermans et al. [Ti]: Given $\mathbf{U}^{n}=\mathcal{P} \mathbf{u}^{n}$ and $p^{n}$, find $\mathbf{u}^{n+1}$ with $\mathbf{u}^{n+1}=0$ on $\Gamma$ and determine $\phi^{n+1}$ so that $\Delta t \nabla \phi^{n+1}=(I-\mathcal{P}) \mathbf{u}^{n+1}$ by solving

$$
\begin{array}{r}
\frac{\mathbf{u}^{n+1}-\mathbf{U}^{n}}{\Delta t}+\mathcal{P}\left(\mathbf{U}^{n} \cdot \nabla \mathbf{U}^{n}-\mathbf{f}^{n}\right)+\nabla p^{n}=\nu \Delta \mathbf{u}^{n+1} \quad \text { in } \Omega, \\
\Delta \phi^{n+1}=\nabla \cdot \mathbf{u}^{n+1} / \Delta t \quad \text { in } \Omega, \quad \mathbf{n} \cdot \nabla \phi^{n+1}=0 \quad \text { on } \Gamma,
\end{array}
$$

and set $\mathbf{U}^{n+1}=\mathbf{u}^{n+1}-\Delta t \nabla \phi^{n+1}=\mathcal{P} \mathbf{u}^{n+1}$ and

$$
p^{n+1}=p^{n}-\nu \nabla \cdot \mathbf{u}^{n+1}+\phi^{n+1} .
$$

This differs from the approach of [Ti] only in the presence of the projection applied to the convection/forcing term. To see that this scheme is like (26) with $\lambda=1 / \Delta t$, apply $I-\mathcal{P}$ to $(27)$ and use Lemma 1 , obtaining

$$
\begin{aligned}
\nabla\left(\phi^{n+1}+p^{n}\right) & =\nu \Delta(I-\mathcal{P}) \mathbf{u}^{n+1}+\nu(\Delta \mathcal{P}-\mathcal{P} \Delta) \mathbf{u}^{n+1} \\
& =\nu \nabla \nabla \cdot \mathbf{u}^{n+1}+\nu \nabla p_{\mathrm{S}}^{n+1} .
\end{aligned}
$$

Using (29) we find $p^{n+1}=\nu p_{\mathrm{S}}^{n+1}$.

This same scheme is also equivalent to the following scheme close to a firstorder version of a scheme proposed in the much-cited work of Kim and Moin $[\mathrm{KM}]$ and examined in the study of numerical boundary layers by Brown et al. $[\mathrm{BCM}]$ : Given $\mathbf{U}^{n}=\mathcal{P} \mathbf{u}^{n}$ and $\phi^{n}$, find $\mathbf{u}^{n+1}$ with $\mathbf{u}^{n+1}=\Delta t \nabla \phi^{n}$ on $\Gamma$, and determine $\phi^{n+1}$ so that $\Delta t \nabla \phi^{n+1}=(I-\mathcal{P}) \mathbf{u}^{n+1}$, by solving

$$
\begin{aligned}
\frac{\mathbf{u}^{n+1}-\mathbf{U}^{n}}{\Delta t} & +\mathcal{P}\left(\mathbf{U}^{n} \cdot \nabla \mathbf{U}^{n}-\mathbf{f}^{n}\right)=\nu \Delta \mathbf{u}^{n+1} \quad \text { in } \Omega, \\
\Delta \phi^{n+1} & =\nabla \cdot \mathbf{u}^{n+1} / \Delta t \quad \text { in } \Omega, \quad \mathbf{n} \cdot \nabla \phi^{n+1}=0 \quad \text { on } \Gamma,
\end{aligned}
$$


and set $\mathbf{U}^{n+1}=\mathbf{u}^{n+1}-\Delta t \nabla \phi^{n+1}=\mathcal{P} \mathbf{u}^{n+1}$. To compare with (26), let

$$
\begin{aligned}
& \mathbf{v}^{n+1}=\mathbf{u}^{n+1}-(I-\mathcal{P}) \mathbf{u}^{n}=\mathbf{u}^{n+1}-\Delta t \nabla \phi^{n}, \\
& p^{n}=\phi^{n}-\nu \Delta t \Delta \phi^{n}=\phi^{n}-\nu \nabla \cdot \mathbf{u}^{n} .
\end{aligned}
$$

Then $\mathcal{P} \mathbf{v}^{n}=\mathcal{P} \mathbf{u}^{n}=\mathbf{U}^{n}$ and using Lemma 1 one finds

$$
\frac{\mathbf{v}^{n+1}-\mathbf{U}^{n}}{\Delta t}+\mathcal{P}\left(\mathbf{U}^{n} \cdot \nabla \mathbf{U}^{n}-\mathbf{f}^{n}\right)+\nabla p^{n}=\nu \Delta \mathbf{v}^{n+1},
$$

with $\mathbf{v}^{n+1}=0$ on $\Gamma$. Applying $I-\mathcal{P}$ to (30) and using (33) we find

$$
\nabla p^{n}=\frac{1}{\Delta t}(I-\mathcal{P}) \mathbf{u}^{n}-\nu \Delta(I-\mathcal{P}) \mathbf{u}^{n}=\nu(\Delta \mathcal{P}-\mathcal{P} \Delta) \mathbf{u}^{n}=\nu \nabla p_{\mathrm{S}}^{n} .
$$

The fact that the scheme of Kim and Moin can be transformed into that of Timmerman et al. has been observed in [GMS].

Finally, if one starts from (26) and retains the linear damping term with $\lambda=1 / \Delta t$ but omits the term $\nabla p_{\mathrm{S}}^{n}$ that produces diffusive damping of the divergence at the continuous level, one arrives at Chorin's original projection method from [Ch1]. Including linear damping with general $\lambda$ and without $\nabla p_{\mathrm{s}}^{n}$ results in a divergence damping strategy related to Baumgarte's method of relaxing constraints in constrained systems of ordinary differential equations $[\mathrm{Bm}]$ - for an approach to incompressible Navier-Stokes flow that combines Baumgarte's stabilization method with a modified penalty regularization, see [Li].

\subsection{Equivalence with a gauge method}

The scheme (4)-(6) is also related, in a less obvious way, to the gauge method proposed and studied by E and Liu [EL] and by Wang and Liu [WL]. The gauge method is motivated by the goal of splitting the pressure and velocity updates, but its form is superficially very different from (4)-(6). At the time-continuous level, the idea is to represent the velocity in the form

$$
\mathbf{u}=\mathbf{a}+\nabla \phi
$$

and impose sufficient equations and boundary conditions on a and $\phi$ to make $\mathbf{u}$ the Navier-Stokes solution. If (35) is the Helmholtz decomposition of a, so that $\nabla \phi=(\mathcal{P}-I) \mathbf{a}$, then $\mathbf{u}$ is divergence-free. However, we can recover the unconstrained formulation (14), for which $\nabla \cdot \mathbf{u}$ is not necessarily zero, with a different choice of the gauge variable $\phi$. Namely, we require

$$
\begin{aligned}
\partial_{t} \mathbf{a}+\mathcal{P}(\mathbf{u} \cdot \nabla \mathbf{u}-\mathbf{f})=\nu \Delta \mathbf{a} & \text { in } \Omega, \\
\mathbf{a}+\nabla \phi=0 & \text { on } \Gamma, \\
\partial_{t} \phi+\nu p_{\mathrm{S}}=\nu \Delta \phi & \text { in } \Omega, \\
\mathbf{n} \cdot \nabla \phi=0 & \text { on } \Gamma,
\end{aligned}
$$

where $p_{\mathrm{S}}$ is the Stokes pressure determined from $\mathbf{u}$ via (21). We recover (14) by adding the gradient of (38) to (36). 
Upon time discretization, one obtains a scheme that can be written in the following form. (See Appendix $\mathrm{C}$ for further details.) Given $\mathbf{a}^{n}$ at time $t_{n}=n \Delta t$ such that $\mathbf{n} \cdot \mathbf{a}^{n}=0$ on $\Gamma$, find $\mathbf{u}^{n}$ and $\phi^{n}$ so that

$$
\begin{aligned}
\mathbf{u}^{n} & =\mathcal{P} \mathbf{a}^{n}+\nu \Delta t(I-\mathcal{P}) \Delta \mathbf{a}^{n}, \\
\Delta \phi^{n} & =-\nabla \cdot \mathbf{a}^{n} \quad \text { in } \Omega, \quad \mathbf{n} \cdot \nabla \phi^{n}=0 \quad \text { on } \Gamma,
\end{aligned}
$$

then determine $\mathbf{a}^{n+1}$ by solving

$$
\begin{array}{rlrl}
\frac{\mathbf{a}^{n+1}-\mathbf{a}^{n}}{\Delta t}+\mathcal{P}\left(\mathbf{u}^{n} \cdot \nabla \mathbf{u}^{n}-\mathbf{f}^{n}\right) & =\nu \Delta \mathbf{a}^{n+1} & & \text { in } \Omega, \\
\mathbf{a}^{n+1}+\nabla \phi^{n} & =0 & \text { on } \Gamma .
\end{array}
$$

The method in [EL, WL] differs only in that $\mathbf{u}^{n}$ in (42) is replaced by $\mathcal{P} \mathbf{u}^{n}$. For a closer comparison, we note that $\nabla \phi^{n}=(\mathcal{P}-I) \mathbf{a}^{n}$ and hence

$$
\mathbf{u}^{n+1}=\mathbf{a}^{n+1}+\nabla \phi^{n} .
$$

Proposition 1 The scheme (4)-(6) is equivalent to the gauge method (40)(43).

For the proof we refer to Appendix C, where we establish equivalence in the general case with nonhomogeneous boundary conditions and a nontrivial divergence constraint.

\subsection{Previous stability and convergence analysis}

We briefly review what has been proven regarding the stability and convergence of schemes related to (4)-(6) that achieve efficiency through a decoupling of the updates of pressure and velocity. In many cases, stability has been studied through normal mode analysis for the linear Stokes equations in periodic strip domains or a half space. Orszag et al. [OID] were perhaps the first to perform such an analysis of a projection method. The works [HP, Pe, JL] treat schemes corresponding to (4)-(6) by this approach, and the gauge method was dealt with in $[\mathrm{EL}]$. As pointed out by Petersson [Pe], showing that the norm of the amplification factor is less than 1 in normal mode analysis establishes only a necessary conditon for stability.

Error estimates that establish a rate of convergence of the scheme have been established for sufficiently smooth solutions of the Stokes or Navier-Stokes equations in a number of cases. We refer to [GMS] for a recent review of results concerning projection methods for Stokes equations in sufficiently smooth domains, related to the methods of Timmermans et al. and Kim and Moin, as well as the schemes of [HP, Pe, GuS, JL]. An analysis of the gauge method was performed by Wang and Liu [WL], who established unconditional stability for the Stokes problem and error estimates for fully discrete Navier-Stokes equations on a staggered grid in a periodic strip.

By comparison, the primary novelty of the present paper concerns stability analysis for approximating strong solutions of the Navier-Stokes equations 
in general smooth $\left(C^{3}\right)$ domains, with the spatially continuous scheme (4)-(6) and with fully discrete finite element methods not requiring any compatibility between velocity and pressure spaces related to an inf-sup condition. We establish convergence to local strong solutions by standard compactness arguments. Rate-of-convergence studies are left for future work.

\section{Estimate on the commutator $\Delta \mathcal{P}-\mathcal{P} \Delta$}

The purpose of this section is to prove Theorem 1 . The proof is rather elementary, based upon (i) an energy partitioning lemma for harmonic functions, that controls the difference between normal and tangential parts of the Dirichlet integral near the boundary (see Lemma 2), and (ii) an orthogonality identity for the Stokes pressure in terms of a part of velocity near and parallel to the boundary.

\subsection{Preliminaries}

Let $\Omega \subset \mathbb{R}^{N}$ be a bounded domain with $C^{3}$ boundary $\Gamma$. For any $x \in \Omega$ we let

$$
\Phi(x)=\operatorname{dist}(x, \Gamma)
$$

denote the distance from $x$ to $\Gamma$. For any $s>0$ we denote the set of points in $\Omega$ within distance $s$ from $\Gamma$ by

$$
\Omega_{s}=\{x \in \Omega \mid \Phi(x)<s\},
$$

and set $\Omega_{s}^{c}=\Omega \backslash \Omega_{s}$ and $\Gamma_{s}=\{x \in \Omega \mid \Phi(x)=s\}$. Since $\Gamma$ is $C^{3}$ and compact, there exists $s_{0}>0$ such that $\Phi$ is $C^{3}$ in $\Omega_{s_{0}}$ and its gradient is a unit vector, with $|\nabla \Phi(x)|=1$ for every $x \in \Omega_{s_{0}}$. We let

$$
\mathbf{n}(x)=-\nabla \Phi(x)
$$

then $\mathbf{n}(x)$ is the outward unit normal to $\Gamma_{s}=\partial \Omega_{s}^{c}$ for $s=\Phi(x)$, and $\mathbf{n} \in$ $C^{2}\left(\bar{\Omega}_{s_{0}}, \mathbb{R}^{N}\right)$.

It suffices to establish the estimate (15) for all $\mathbf{u} \in C^{2}\left(\bar{\Omega}, \mathbb{R}^{N}\right)$ with $\mathbf{u}=$ 0 on $\Gamma$, since the space of such functions is dense in $H^{2} \cap H_{0}^{1}\left(\Omega, \mathbb{R}^{N}\right)$. This fact is a consequence of $C^{2, \alpha}$ elliptic regularity theory [GT], but we indicate a more elementary argument in keeping with the nature of the rest of this section: Let $B(0, r)=\left\{x \in \mathbb{R}^{N}|| x \mid<r\right\}$ and $\mathbb{R}_{+}^{N}=\left\{x \mid x_{N}>0\right\}$. By a standard argument using partition of unity and $C^{2}$ boundary-flattening maps, the problem is reduced to one of local approximation for locally flat boundary. It suffices to show that if $r_{0}>r_{1}>0$ and $B\left(0, r_{0}\right) \cap \Omega=B\left(0, r_{0}\right) \cap \mathbb{R}_{+}^{N}$, then any $u \in H^{2} \cap H_{0}^{1}(\Omega)$ that is supported in $B\left(0, r_{1}\right)$ can be approximated by functions $u_{k} \in C^{2}\left(\mathbb{R}^{N}\right)$ that vanish when $x_{N}=0$ and when $|x|>r_{0}$. Fix a smooth radial function $\xi$ on $\mathbb{R}^{N}$ with $\xi(x)=1$ for $|x|<r_{1}, \xi(x)=0$ for $|x|>r_{0}$. Given $u$ as described, then $f=(I-\Delta) u \in L^{2}(\Omega)$ and $f=0$ outside $B\left(0, r_{1}\right)$. Approximate $f$ in $L^{2}$ norm by smooth $f_{k}$ compactly supported in $B\left(0, r_{1}\right) \cap \mathbb{R}_{+}^{N}$ and extend 
$f_{k}$ to $\mathbb{R}^{N}$ as odd in $x_{N}$ and zero for $|x|>r_{1}$. Now $u_{k}=\xi(I-\Delta)^{-1} f_{k}$ is smooth in $\mathbb{R}^{N}$ and vanishes when $|x|>r_{0}$, is odd in $x_{N}$ so vanishes when $x_{N}=0$, and converges to $u=\xi(I-\Delta)^{-1} f$ in $H^{2}(\Omega)$.

\subsection{Energy partitioning lemma}

Our proof of Theorem 1 crucially involves a comparison between the normal and tangential parts of the Dirichlet integral for harmonic functions in the tubular domains $\Omega_{s}$ for small $s>0$.

Lemma 2 Let $\Omega$ be a bounded domain with $C^{2}$ boundary and suppose $\Phi$ is $C^{2}$ in $\Omega_{s_{0}}, s_{0}>0$. Then there exists $C_{0} \geq 0$ such that whenever $p$ is a harmonic function in $\Omega_{s_{0}}$ and $0<s<s_{0}$ we have

$$
\left|\int_{\Omega_{s}}\left(|\mathbf{n} \cdot \nabla p|^{2}-\left|\left(I-\mathbf{n n}^{T}\right) \nabla p\right|^{2}\right)\right| \leq C_{0} s \int_{\Omega_{s_{0}}}|\nabla p|^{2} .
$$

Proof: We let

$$
Q_{0}=|\mathbf{n} \cdot \nabla p|^{2}-\left|\left(I-\mathbf{n n}^{T}\right) \nabla p\right|^{2}=2|\mathbf{n} \cdot \nabla p|^{2}-|\nabla p|^{2},
$$

and note that due to $\mathbf{n}=-\nabla \Phi$ and $|\mathbf{n}|^{2}=1$ we have

$$
Q_{0}=\nabla \Phi \cdot \mathbf{v}, \quad \mathbf{v}=2 \nabla p(\nabla \Phi \cdot \nabla p)-\nabla \Phi|\nabla p|^{2} .
$$

Following a technique used by Sanni on p. 13 of [Sa], we integrate by parts for $0<s<s_{0}$ to obtain

$$
\int_{\Omega_{s}} Q_{0}=\int_{\Gamma_{s}} \Phi(-\mathbf{n} \cdot \mathbf{v})-\int_{\Omega_{s}} \Phi \nabla \cdot \mathbf{v}=s \int_{\Gamma_{s}} Q_{0}+\int_{\Omega_{s}} \Phi Q_{1},
$$

where, since $\Delta p=0$ (and with summation on repeated indices implied),

$$
\begin{aligned}
Q_{1}=-\nabla \cdot \mathbf{v} & =-2 \nabla^{2} \Phi:(\nabla p \otimes \nabla p)+(\Delta \Phi)|\nabla p|^{2} \\
& =-2\left(\partial_{i} \partial_{j} \Phi\right)\left(\partial_{i} p\right)\left(\partial_{j} p\right)+\left(\partial_{i}^{2} \Phi\right)\left(\partial_{j} p\right)^{2} .
\end{aligned}
$$

We have $\left|Q_{0}\right| \leq|\nabla p|^{2}$ and $\left|Q_{1}\right| \leq C_{1}|\nabla p|^{2}$ where $C_{1}$ depends only upon $\Omega$ and $s_{0}$. We divide (47) by $s^{2}$ and integrate in $s$ to obtain

$$
\begin{aligned}
\frac{1}{s} \int_{\Omega_{s}} Q_{0}-\frac{1}{s_{0}} \int_{\Omega_{s_{0}}} Q_{0} & =\int_{s}^{s_{0}} \frac{1}{r^{2}}\left(\int_{\Omega_{r}} \Phi Q_{1}\right) d r \\
& =\frac{1}{s} \int_{\Omega_{s}} \Phi Q_{1}-\frac{1}{s_{0}} \int_{\Omega_{s_{0}}} \Phi Q_{1}+\int_{\Omega_{s_{0}} \backslash \Omega_{s}} Q_{1}
\end{aligned}
$$

Since $|\Phi(x)| \leq r$ in $\Omega_{r}$, it follows directly that

$$
\left|\int_{\Omega_{s}} Q_{0}\right| \leq s \int_{\Omega_{s_{0}}}\left(s_{0}^{-1}\left|Q_{0}\right|+2\left|Q_{1}\right|\right) \leq C_{0} s \int_{\Omega_{s_{0}}}|\nabla p|^{2}
$$

with $C_{0}=s_{0}^{-1}+2 C_{1}$. 


\subsection{Boundary identities}

As a consequence of the no-slip boundary condition $\mathbf{u}=0$ on $\Gamma$, we obtain two key identities that involve the parts of $\mathbf{u}$ parallel and normal to the boundary.

Lemma 3 Let $\Omega \subset \mathbb{R}^{N}$ be a bounded domain with boundary $\Gamma$ of class $C^{2}$. Let $\mathbf{u} \in C^{1}\left(\bar{\Omega}, \mathbb{R}^{N}\right)$ and suppose that

$$
\mathbf{u}_{\|}=\left(I-\mathbf{n} \mathbf{n}^{T}\right) \mathbf{u}, \quad \mathbf{u}_{\perp}=\mathbf{n} \mathbf{n}^{T} \mathbf{u}
$$

in some neighborhood of $\Gamma$. Then the following are valid:

(i) If $\mathbf{u}=0$ on $\Gamma$ then $\nabla \cdot \mathbf{u}_{\|}=0$ on $\Gamma$.

(ii) If $\mathbf{n} \cdot \mathbf{u}=0$ on $\Gamma$ then $\nabla \mathbf{u}_{\perp}-\nabla \mathbf{u}_{\perp}{ }^{T}=0$ on $\Gamma$.

(In particular, $\nabla \times \mathbf{u}_{\perp}=0$ on $\Gamma$ if $N=2$ or 3 .)

Proof: To begin, recall $\mathbf{n}=-\nabla \Phi$. Equality of mixed partial derivatives yields $\partial_{j} n_{i}=\partial_{i} n_{j}$ for all $i, j=1, \ldots, N$. Together with the fact $n_{i} n_{i}=1$, we infer that for small $s>0$, throughout $\Omega_{s}$ we have

$$
n_{i} \partial_{j} n_{i}=0 \quad \text { and } \quad n_{i} \partial_{i} n_{j}=0 .
$$

(i) First, for any $f \in C^{1}(\bar{\Omega})$, if $f=0$ on $\Gamma$ then $\nabla f \| \mathbf{n}$ on $\Gamma$, which means

$$
\left(I-\mathbf{n n}^{T}\right) \nabla f=0, \quad \text { or } \quad\left(\partial_{k}-n_{k} n_{j} \partial_{j}\right) f=0 \quad \text { for } k=1, \ldots, N .
$$

Now suppose $\mathbf{u} \in C^{2}\left(\bar{\Omega}, \mathbb{R}^{N}\right)$ with $\mathbf{u}=0$ on $\Gamma$. Then, after taking derivatives in $\Omega_{s}$ for some $s>0$ and then taking the trace on $\Gamma$, using (49) we get

$$
\nabla \cdot\left(\left(I-\mathbf{n n}^{T}\right) \mathbf{u}\right)=\partial_{j}\left(u_{j}-n_{j} n_{k} u_{k}\right)=\partial_{j} u_{j}-n_{j} n_{k} \partial_{j} u_{k}=\partial_{j} u_{j}-\partial_{k} u_{k}=0 .
$$

(ii) Let $f=\mathbf{n} \cdot \mathbf{u}$ so that $\mathbf{u}_{\perp}=\mathbf{n} f$. Using $\partial_{j} n_{i}=\partial_{i} n_{j}$ and (49) we find that

$$
\partial_{j}\left(n_{i} f\right)-\partial_{i}\left(n_{j} f\right)=n_{i} n_{j} n_{k} \partial_{k} f-n_{j} n_{i} n_{k} \partial_{k} f=0 .
$$

This proves (ii). If $N=2$ or 3 this just means $\nabla \times(\mathbf{n} f)=\mathbf{n} \times \nabla f=0$ on $\Gamma$.

\subsection{Orthogonality identity}

Let $\mathbf{u} \in C^{2}\left(\bar{\Omega}, \mathbb{R}^{N}\right)$. As indicated in (25), in two and three dimensions the Stokes pressure vanishes if the vorticity tangential to the boundary vanishes. In any dimension $N \geq 2$, the Stokes pressure $p_{\mathrm{S}}$ from (20) is determined by

$$
\begin{aligned}
\int_{\Omega} \nabla p_{\mathrm{S}} \cdot \nabla \phi & =\int_{\Omega}\left(\partial_{j}^{2} u_{i}-\partial_{i} \partial_{j} u_{j}\right) \partial_{i} \phi=\int_{\Gamma} n_{j}\left(\partial_{j} u_{i}-\partial_{i} u_{j}\right) \partial_{i} \phi \\
& =\int_{\Gamma} \mathbf{n} \cdot\left(\nabla \mathbf{u}-\nabla \mathbf{u}^{T}\right) \nabla \phi \quad \forall \phi \in H^{1}(\Omega) .
\end{aligned}
$$

Thus, $p_{\mathrm{S}}$ is not affected by any part of the velocity field that contributes nothing to $\mathbf{n} \cdot\left(\nabla \mathbf{u}-\nabla \mathbf{u}^{T}\right)$. This means the Stokes pressure is not affected by the part of 
the velocity field in the interior of $\Omega$, nor is it affected by the normal component of velocity near the boundary if $\left.\mathbf{n} \cdot \mathbf{u}\right|_{\Gamma}=0$, by Lemma 3 (ii).

This motivates us to focus on the part of velocity near and parallel to the boundary. We make the decomposition

$$
\begin{gathered}
\mathbf{u}=\mathbf{u}_{\perp}+\mathbf{u}_{\|} \\
\mathbf{u}_{\perp}=\xi \mathbf{n n}^{T} \mathbf{u}+(1-\xi) \mathbf{u}, \quad \mathbf{u}_{\|}=\xi\left(I-\mathbf{n n}^{T}\right) \mathbf{u}
\end{gathered}
$$

where $\xi$ is a cutoff function satisfying $\xi(x)=1$ when $\Phi(x)<\frac{1}{2} s$ and $\xi(x)=0$ when $\Phi(x) \geq s$. We can define $\xi$ as follows. Let $\rho:[0, \infty) \rightarrow[0,1]$ be a smooth decreasing function with $\rho(t)=1$ for $t<\frac{1}{2}$ and $\rho(t)=0$ for $t \geq 1$. With $\xi(x)=\rho(\Phi(x) / s), \xi$ is $C^{3}$ for small $s>0$ and has the desired properties.

Suppose that $\mathbf{u}=0$ on $\Gamma$. Since then $\mathbf{u}_{\perp} \in C^{2}\left(\bar{\Omega}, \mathbb{R}^{N}\right)$ with $\mathbf{u}_{\perp}=0$ and $\mathbf{n} \cdot\left(\nabla \mathbf{u}_{\perp}-\nabla \mathbf{u}_{\perp}^{T}\right)=0$ on $\Gamma$, we have that the Stokes pressure for $\mathbf{u}_{\perp}$ vanishes - replacing $\mathbf{u}$ by $\mathbf{u}_{\perp}$ in (19) and (20) and (50) we get

$$
(\Delta \mathcal{P}-\mathcal{P} \Delta) \mathbf{u}_{\perp}=(I-\mathcal{P})(\Delta-\nabla \nabla \cdot) \mathbf{u}_{\perp}=0 .
$$

With (51), this proves

$$
\nabla p_{\mathrm{S}}=(\Delta \mathcal{P}-\mathcal{P} \Delta) \mathbf{u}=(\Delta \mathcal{P}-\mathcal{P} \Delta) \mathbf{u}_{\|}=(I-\mathcal{P})(\Delta-\nabla \nabla \cdot) \mathbf{u}_{\|}
$$

Lemma 4 Let $\Omega \subset \mathbb{R}^{N}$ be a bounded domain with $C^{3}$ boundary, and let $\mathbf{u} \in$ $H^{2} \cap H_{0}^{1}\left(\Omega, \mathbb{R}^{N}\right)$. Let $p_{S}$ and $\mathbf{u}_{\|}$be defined via (19) and (52) respectively. Then for any $q \in H^{1}(\Omega)$ that satisfies $\Delta q=0$ in the sense of distributions,

$$
\left\langle\Delta \mathbf{u}_{\|}-\nabla p_{S}, \nabla q\right\rangle=0 .
$$

In particular we can let $q=p_{S}$, so $\left\langle\Delta \mathbf{u}_{\|}-\nabla p_{S}, \nabla p_{S}\right\rangle=0$ and

$$
\left\|\Delta \mathbf{u}_{\|}\right\|^{2}=\left\|\Delta \mathbf{u}_{\|}-\nabla p_{S}\right\|^{2}+\left\|\nabla p_{S}\right\|^{2} .
$$

Proof: It suffices to suppose $\mathbf{u} \in C^{2}\left(\bar{\Omega}, \mathbb{R}^{N}\right)$ with $\mathbf{u}=0$ on $\Gamma$ by the density remark in section 3.1. By Lemma 3(i),

$$
\left.\nabla \cdot \mathbf{u}_{\|}\right|_{\Gamma}=0
$$

so $\nabla \cdot \mathbf{u}_{\|} \in H_{0}^{1}(\Omega)$, thus $\left\langle\nabla \nabla \cdot \mathbf{u}_{\|}, \nabla q\right\rangle=-\left\langle\nabla \cdot \mathbf{u}_{\|}, \Delta q\right\rangle=0$. Now (54) entails

$$
\left\langle\nabla p_{\mathrm{S}}, \nabla q\right\rangle=\left\langle\Delta \mathbf{u}_{\|}, \nabla q\right\rangle
$$

This proves (55). Then (56) follows by $L^{2}$-orthogonality.

\subsection{Proof of Theorem 1}

Let $\varepsilon>0$ and $\beta=\frac{1}{2}+\varepsilon$. Fix $\beta_{1}<1$ such that $1+\varepsilon_{0}:=\beta\left(1+\beta_{1}\right)>1$, and fix $s, \varepsilon_{1}, \varepsilon_{2}>0$ small so that $2 \varepsilon_{1}<\varepsilon_{0}$ and $1-\varepsilon_{2}-2 C_{0} s>\beta_{1}$ with $C_{0}$ as in Lemma 2. 
Let $\mathbf{u} \in H^{2} \cap H_{0}^{1}\left(\Omega, \mathbb{R}^{N}\right)$, define the Stokes pressure $\nabla p_{\mathrm{S}}=(\Delta \mathcal{P}-\mathcal{P} \Delta) \mathbf{u}$, and make the decomposition $\mathbf{u}=\mathbf{u}_{\perp}+\mathbf{u}_{\|}$as in the previous subsection. Then by Lemma 4 we have

$$
\|\Delta \mathbf{u}\|^{2}=\left\|\Delta \mathbf{u}_{\perp}\right\|^{2}+2\left\langle\Delta \mathbf{u}_{\perp}, \Delta \mathbf{u}_{\|}\right\rangle+\left\|\Delta \mathbf{u}_{\|}-\nabla p_{\mathrm{S}}\right\|^{2}+\left\|\nabla p_{\mathrm{S}}\right\|^{2} .
$$

We will establish the Theorem with the help of two further estimates.

Claim 1: There exists a constant $C_{1}>0$ independent of $\mathbf{u}$ such that

$$
\left\langle\Delta \mathbf{u}_{\perp}, \Delta \mathbf{u}_{\|}\right\rangle \geq-\varepsilon_{1}\|\Delta \mathbf{u}\|^{2}-C_{1}\|\nabla \mathbf{u}\|^{2} .
$$

Claim 2: There exists a constant $C_{2}$ independent of $\mathbf{u}$ such that

$$
\left\|\Delta \mathbf{u}_{\|}-\nabla p_{\mathrm{S}}\right\|^{2} \geq \beta_{1}\left\|\nabla p_{\mathrm{S}}\right\|^{2}-C_{2}\|\nabla \mathbf{u}\|^{2}
$$

Combining the two claims with (59), we get

$$
\left(1+2 \varepsilon_{1}\right)\|\Delta \mathbf{u}\|^{2} \geq\left(1+\beta_{1}\right)\left\|\nabla p_{\mathrm{S}}\right\|^{2}-\left(C_{2}+2 C_{1}\right)\|\nabla \mathbf{u}\|^{2} .
$$

Multiplying by $\beta$ and using $\beta\left(1+\beta_{1}\right)=1+\varepsilon_{0}>1+2 \varepsilon_{1}$ yields (15).

Proof of claim 1: From the definitions in (52), we have

$$
\Delta \mathbf{u}_{\perp}=\xi \mathbf{n n}^{T} \Delta \mathbf{u}+(1-\xi) \Delta \mathbf{u}+R_{1}, \quad \Delta \mathbf{u}_{\|}=\xi\left(I-\mathbf{n n}^{T}\right) \Delta \mathbf{u}+R_{2},
$$

where $\left\|R_{1}\right\|+\left\|R_{2}\right\| \leq C\|\nabla \mathbf{u}\|$ with $C$ independent of $\mathbf{u}$. Since $I-\mathbf{n n}^{T}=$ $\left(I-\mathbf{n} \mathbf{n}^{T}\right)^{2}$,

$$
\left(\xi \mathbf{n} \mathbf{n}^{T} \Delta \mathbf{u}+(1-\xi) \Delta \mathbf{u}\right) \cdot\left(\xi\left(I-\mathbf{n} \mathbf{n}^{T}\right) \Delta \mathbf{u}\right)=0+\xi(1-\xi)\left|\left(I-\mathbf{n n}^{T}\right) \Delta \mathbf{u}\right|^{2} \geq 0
$$

This means the leading term of $\left\langle\Delta \mathbf{u}_{\perp}, \Delta \mathbf{u}_{\|}\right\rangle$is non-negative. Using the inequality $|\langle a, b\rangle| \leq\left(\varepsilon_{1} / C\right)\|a\|^{2}+\left(C / \varepsilon_{1}\right)\|b\|^{2}$ and the bounds on $R_{1}$ and $R_{2}$ to estimate the remaining terms, it is easy to obtain $(60)$.

Proof of claim 2: Let $\mathbf{a}=\nabla p_{\mathrm{S}}$ and $\mathbf{b}=\Delta \mathbf{u}_{\|}$, and put

(64) $\mathbf{a}_{\|}=\left(I-\mathbf{n} \mathbf{n}^{T}\right) \mathbf{a}, \quad \mathbf{a}_{\perp}=\left(\mathbf{n n}^{T}\right) \mathbf{a}, \quad \mathbf{b}_{\|}=\left(I-\mathbf{n n}^{T}\right) \mathbf{b}, \quad \mathbf{b}_{\perp}=\left(\mathbf{n n}^{T}\right) \mathbf{b}$.

Recall $\mathbf{u}_{\|}$is supported in $\Omega_{s}=\{x \in \Omega \mid \Phi(x)<s\}$. Due to (63), we have

$$
\int_{\Omega_{s}}\left|\mathbf{b}_{\perp}\right|^{2}=\int_{\Omega_{s}}\left|\mathbf{n} \cdot \Delta \mathbf{u}_{\|}\right|^{2}=\int_{\Omega_{s}}\left|\mathbf{n} \cdot R_{2}\right|^{2} \leq C \int_{\Omega}|\nabla \mathbf{u}|^{2}
$$

Since $\mathbf{b}=0$ in $\Omega_{s}^{c}=\{x \in \Omega \mid \Phi(x) \geq s\}$, we have

(66) $\left\|\Delta \mathbf{u}_{\|}-\nabla p_{\mathrm{S}}\right\|^{2}=\int_{\Omega}|\mathbf{a}-\mathbf{b}|^{2}=\int_{\Omega_{s}^{c}}|\mathbf{a}|^{2}+\int_{\Omega_{s}}\left|\mathbf{a}_{\perp}-\mathbf{b}_{\perp}\right|^{2}+\int_{\Omega_{s}}\left|\mathbf{a}_{\|}-\mathbf{b}_{\|}\right|^{2}$.

We estimate the terms in (66) as follows. First,

(67) $\int_{\Omega_{s}}\left|\mathbf{a}_{\perp}-\mathbf{b}_{\perp}\right|^{2} \geq \int_{\Omega_{s}}\left(\left|\mathbf{a}_{\perp}\right|^{2}-2 \mathbf{a}_{\perp} \cdot \mathbf{b}_{\perp}\right) \geq\left(1-\varepsilon_{2}\right) \int_{\Omega_{s}}\left|\mathbf{a}_{\perp}\right|^{2}-\frac{1}{\varepsilon_{2}} \int_{\Omega_{s}}\left|\mathbf{b}_{\perp}\right|^{2}$. 
Due to the orthogonality in Lemma 4 , we have $\langle\mathbf{a}, \mathbf{a}-\mathbf{b}\rangle=0$, hence

(68) $0=\int_{\Omega} \mathbf{a} \cdot(\mathbf{a}-\mathbf{b})=\int_{\Omega_{s}^{c}}|\mathbf{a}|^{2}+\int_{\Omega_{s}} \mathbf{a}_{\perp} \cdot\left(\mathbf{a}_{\perp}-\mathbf{b}_{\perp}\right)+\int_{\Omega_{s}} \mathbf{a}_{\|} \cdot\left(\mathbf{a}_{\|}-\mathbf{b}_{\|}\right)$.

For a sharp estimate we need to treat $\mathbf{b}_{\|}$carefully. Using (68) we obtain

$$
\begin{aligned}
\int_{\Omega_{s}}\left|\mathbf{a}_{\|}-\mathbf{b}_{\|}\right|^{2}+\left|\mathbf{a}_{\|}\right|^{2} & \geq-2 \int_{\Omega_{s}} \mathbf{a}_{\|} \cdot\left(\mathbf{a}_{\|}-\mathbf{b}_{\|}\right) \\
& =2 \int_{\Omega_{s}^{c}}|\mathbf{a}|^{2}+2 \int_{\Omega_{s}} \mathbf{a}_{\perp} \cdot\left(\mathbf{a}_{\perp}-\mathbf{b}_{\perp}\right) \\
& \geq 2 \int_{\Omega_{s}^{c}}|\mathbf{a}|^{2}+\left(2-\varepsilon_{2}\right) \int_{\Omega_{s}}\left|\mathbf{a}_{\perp}\right|^{2}-\frac{1}{\varepsilon_{2}} \int_{\Omega_{s}}\left|\mathbf{b}_{\perp}\right|^{2}
\end{aligned}
$$

hence

(69) $\int_{\Omega_{s}}\left|\mathbf{a}_{\|}-\mathbf{b}_{\|}\right|^{2} \geq\left(1-\varepsilon_{2}\right) \int_{\Omega_{s}}\left|\mathbf{a}_{\|}\right|^{2}+\left(2-\varepsilon_{2}\right) \int_{\Omega_{s}}\left(\left|\mathbf{a}_{\perp}\right|^{2}-\left|\mathbf{a}_{\|}\right|^{2}\right)-\frac{1}{\varepsilon_{2}} \int_{\Omega_{s}}\left|\mathbf{b}_{\perp}\right|^{2}$.

Using (67) and (69) in (66) yields

(70) $\int_{\Omega}|\mathbf{a}-\mathbf{b}|^{2} \geq\left(1-\varepsilon_{2}\right) \int_{\Omega}|\mathbf{a}|^{2}+\left(2-\varepsilon_{2}\right) \int_{\Omega_{s}}\left(\left|\mathbf{a}_{\perp}\right|^{2}-\left|\mathbf{a}_{\|}\right|^{2}\right)-\frac{2}{\varepsilon_{2}} \int_{\Omega_{s}}\left|\mathbf{b}_{\perp}\right|^{2}$.

Finally, using Lemma 2 and the estimate (65) we infer

$$
\int_{\Omega}\left|\nabla p_{\mathrm{S}}-\Delta \mathbf{u}_{\|}\right|^{2} \geq\left(1-\varepsilon_{2}-2 C_{0} s\right) \int_{\Omega}\left|\nabla p_{\mathrm{S}}\right|^{2}-C \int_{\Omega}|\nabla \mathbf{u}|^{2} .
$$

This establishes Claim 2, and finishes the proof of Theorem 1.

Note that estimate (15) also holds with $\int_{\Omega}|\nabla \mathbf{u}|^{2}$ replaced by $\int_{\Omega}|\mathbf{u}|^{2}$, due to a simple interpolation estimate.

\section{Unconditional stability of time discretization with pressure explicit}

In this section we exploit Theorem 1 to establish the unconditional stability of the simple time discretization scheme (4)-(6) for the initial-boundary-value problem for (11)-(13), our unconstrained formulation of the Navier-Stokes equations with no-slip boundary conditions, with initial conditions

$$
\mathbf{u}=\mathbf{u}_{\text {in }} \quad \text { in } \Omega \text { for } t=0 .
$$

We focus here on the case of two and three dimensions.

We assume $\mathbf{u}_{\text {in }} \in H_{0}^{1}\left(\Omega, \mathbb{R}^{N}\right)$ and $\mathbf{f} \in L^{2}\left(0, T_{0} ; L^{2}\left(\Omega, \mathbb{R}^{N}\right)\right)$ for some given $T_{0}>0$. We consider the time-discrete scheme (4)-(6) with

$$
\mathbf{f}^{n}=\frac{1}{\Delta t} \int_{n \Delta t}^{(n+1) \Delta t} \mathbf{f}(t) d t
$$


and take $\mathbf{u}^{0} \in H^{2} \cap H_{0}^{1}\left(\Omega, \mathbb{R}^{N}\right)$ to approximate $\mathbf{u}_{\text {in }}$ in $H_{0}^{1}\left(\Omega, \mathbb{R}^{N}\right)$.

Let us begin making estimates - our main result is stated as Theorem 2 below. Let $\nabla p_{\mathrm{S}}^{n}=(\Delta \mathcal{P}-\mathcal{P} \Delta) \mathbf{u}^{n}$ be the Stokes pressure for $\mathbf{u}^{n}$, so that by (20),

$$
\int_{\Omega} \nabla p_{\mathrm{S}}^{n} \cdot \nabla \phi=\int_{\Omega}\left(\Delta \mathbf{u}^{n}-\nabla \nabla \cdot \mathbf{u}^{n}\right) \cdot \nabla \phi \quad \forall \phi \in H^{1}(\Omega) .
$$

Combining this with (4) and taking $\phi=p^{n}$, we obtain the estimate

$$
\left\|\nabla p^{n}\right\| \leq\left\|\mathbf{f}^{n}-\mathbf{u}^{n} \cdot \nabla \mathbf{u}^{n}\right\|+\nu\left\|\nabla p_{\mathrm{S}}^{n}\right\| .
$$

Now $\operatorname{dot}(5)$ with $-\Delta \mathbf{u}^{n+1}$ to obtain

(76) $\frac{1}{2 \Delta t}\left(\left\|\nabla \mathbf{u}^{n+1}\right\|^{2}-\left\|\nabla \mathbf{u}^{n}\right\|^{2}+\left\|\nabla \mathbf{u}^{n+1}-\nabla \mathbf{u}^{n}\right\|^{2}\right)+\nu\left\|\Delta \mathbf{u}^{n+1}\right\|^{2}$

$$
\begin{aligned}
& \leq\left\|\Delta \mathbf{u}^{n+1}\right\|\left(2\left\|\mathbf{f}^{n}-\mathbf{u}^{n} \cdot \nabla \mathbf{u}^{n}\right\|+\nu\left\|\nabla p_{\mathrm{S}}^{n}\right\|\right) \\
& \leq \frac{\varepsilon_{1}}{2}\left\|\Delta \mathbf{u}^{n+1}\right\|^{2}+\frac{2}{\varepsilon_{1}}\left\|\mathbf{f}^{n}-\mathbf{u}^{n} \cdot \nabla \mathbf{u}^{n}\right\|^{2}+\frac{\nu}{2}\left(\left\|\Delta \mathbf{u}^{n+1}\right\|^{2}+\left\|\nabla p_{\mathrm{S}}^{n}\right\|^{2}\right)
\end{aligned}
$$

for any $\varepsilon_{1}>0$. This gives

$$
\begin{gathered}
\frac{1}{\Delta t}\left(\left\|\nabla \mathbf{u}^{n+1}\right\|^{2}-\left\|\nabla \mathbf{u}^{n}\right\|^{2}\right)+\left(\nu-\varepsilon_{1}\right)\left\|\Delta \mathbf{u}^{n+1}\right\|^{2} \\
\leq \frac{8}{\varepsilon_{1}}\left(\left\|\mathbf{f}^{n}\right\|^{2}+\left\|\mathbf{u}^{n} \cdot \nabla \mathbf{u}^{n}\right\|^{2}\right)+\nu\left\|\nabla p_{\mathrm{S}}^{n}\right\|^{2} .
\end{gathered}
$$

Fix any $\beta$ with $\frac{1}{2}<\beta<1$. By Theorem 1 one has

$$
\nu\left\|\nabla p_{\mathrm{S}}^{n}\right\|^{2} \leq \nu \beta\left\|\Delta \mathbf{u}^{n}\right\|^{2}+\nu C_{\beta}\left\|\nabla \mathbf{u}^{n}\right\|^{2} .
$$

Using this in (77), one obtains

$$
\begin{aligned}
\frac{1}{\Delta t}\left(\left\|\nabla \mathbf{u}^{n+1}\right\|^{2}-\right. & \left.\left\|\nabla \mathbf{u}^{n}\right\|^{2}\right)+\left(\nu-\varepsilon_{1}\right)\left(\left\|\Delta \mathbf{u}^{n+1}\right\|^{2}-\left\|\Delta \mathbf{u}^{n}\right\|^{2}\right) \\
& +\left(\nu-\varepsilon_{1}-\nu \beta\right)\left\|\Delta \mathbf{u}^{n}\right\|^{2} \\
& \leq \frac{8}{\varepsilon_{1}}\left(\left\|\mathbf{f}^{n}\right\|^{2}+\left\|\mathbf{u}^{n} \cdot \nabla \mathbf{u}^{n}\right\|^{2}\right)+\nu C_{\beta}\left\|\nabla \mathbf{u}^{n}\right\|^{2} .
\end{aligned}
$$

At this point there are no remaining difficulties with controlling the pressure. It remains only to use the viscosity to control the nonlinear term. We focus on the physically most interesting cases $N=2$ and 3 . We make use of Ladyzhenskaya's inequalities [La]

$$
\begin{array}{ll}
\int_{\mathbb{R}^{N}} g^{4} \leq 2\left(\int_{\mathbb{R}^{N}} g^{2}\right)\left(\int_{\mathbb{R}^{N}}|\nabla g|^{2}\right) & (N=2), \\
\int_{\mathbb{R}^{N}} g^{4} \leq 4\left(\int_{\mathbb{R}^{N}} g^{2}\right)^{1 / 2}\left(\int_{\mathbb{R}^{N}}|\nabla g|^{2}\right)^{3 / 2} & (N=3),
\end{array}
$$


valid for $g \in H^{1}\left(\mathbb{R}^{N}\right)$ with $N=2$ and 3 respectively, together with the fact that the standard bounded extension operator $H^{1}(\Omega) \rightarrow H^{1}\left(\mathbb{R}^{N}\right)$ is also bounded in $L^{2}$ norm, to infer that for all $g \in H^{1}(\Omega)$,

$$
\begin{array}{lr}
\|g\|_{L^{4}}^{2} \leq C\|g\|_{L^{2}}\|g\|_{H^{1}} & (N=2), \\
\|g\|_{L^{3}}^{2} \leq\|g\|_{L^{2}}^{2 / 3}\|g\|_{L^{4}}^{4 / 3} \leq C\|g\|_{L^{2}}\|g\|_{H^{1}} & (N=3) .
\end{array}
$$

Using that $H^{1}(\Omega)$ embeds into $L^{4}$ and $L^{6}$, these inequalities lead to the estimates

$$
\int_{\Omega}\left|\mathbf{u}^{n} \cdot \nabla \mathbf{u}^{n}\right|^{2} \leq \begin{cases}\left\|\mathbf{u}^{n}\right\|_{L^{4}}^{2}\left\|\nabla \mathbf{u}^{n}\right\|_{L^{4}}^{2} \leq C\|\mathbf{u}\|_{L^{2}}\left\|\nabla \mathbf{u}^{n}\right\|_{L^{2}}^{2}\left\|\nabla \mathbf{u}^{n}\right\|_{H^{1}} & (N=2), \\ \left\|\mathbf{u}^{n}\right\|_{L^{6}}^{2}\left\|\nabla \mathbf{u}^{n}\right\|_{L^{3}}^{2} \leq C\left\|\nabla \mathbf{u}^{n}\right\|_{L^{2}}^{3}\left\|\nabla \mathbf{u}^{n}\right\|_{H^{1}} & (N=3) .\end{cases}
$$

By the elliptic regularity estimate $\|\nabla \mathbf{u}\|_{H^{1}} \leq\|\mathbf{u}\|_{H^{2}} \leq C\|\Delta \mathbf{u}\|$, we conclude

$$
\left\|\mathbf{u}^{n} \cdot \nabla \mathbf{u}^{n}\right\|^{2} \leq \begin{cases}\varepsilon_{2}\left\|\Delta \mathbf{u}^{n}\right\|^{2}+4 C \varepsilon_{2}^{-1}\left\|\mathbf{u}^{n}\right\|^{2}\left\|\nabla \mathbf{u}^{n}\right\|^{4} & (N=2) \\ \varepsilon_{2}\left\|\Delta \mathbf{u}^{n}\right\|^{2}+4 C \varepsilon_{2}^{-1}\left\|\nabla \mathbf{u}^{n}\right\|^{6} & (N=2 \text { or } 3)\end{cases}
$$

for any $\varepsilon_{2}>0$. Plug this into (79) and take $\varepsilon_{1}, \varepsilon_{2}>0$ satisfying $\nu-\varepsilon_{1}>0$ and $\varepsilon:=\nu-\varepsilon_{1}-\nu \beta-8 \varepsilon_{2} / \varepsilon_{1}>0$. We get

$$
\begin{aligned}
& \frac{1}{\Delta t}\left(\left\|\nabla \mathbf{u}^{n+1}\right\|^{2}-\left\|\nabla \mathbf{u}^{n}\right\|^{2}\right)+\left(\nu-\varepsilon_{1}\right)\left(\left\|\Delta \mathbf{u}^{n+1}\right\|^{2}-\left\|\Delta \mathbf{u}^{n}\right\|^{2}\right)+\varepsilon\left\|\Delta \mathbf{u}^{n}\right\|^{2} \\
& \quad \leq \frac{8}{\varepsilon_{1}}\left\|\mathbf{f}^{n}\right\|^{2}+\frac{32 C}{\varepsilon_{1} \varepsilon_{2}}\left\|\nabla \mathbf{u}^{n}\right\|^{6}+\nu C_{\beta}\left\|\nabla \mathbf{u}^{n}\right\|^{2}
\end{aligned}
$$

A simple discrete Gronwall-type argument leads to our main stability result:

Theorem 2 Let $\Omega$ be a bounded domain in $\mathbb{R}^{N}(N=2$ or 3$)$ with $C^{3}$ boundary, and let $\nu, M_{0}>0$. Then there exist positive constants $T_{*}$ and $C_{3}$ such that, if $\mathbf{f} \in L^{2}\left(0, T_{0} ; L^{2}\left(\Omega, \mathbb{R}^{N}\right)\right)$ for some $T_{0}>0$ and $\mathbf{u}^{0} \in H_{0}^{1} \cap H^{2}\left(\Omega, \mathbb{R}^{N}\right)$ with

$$
\left\|\nabla \mathbf{u}^{0}\right\|^{2}+\nu \Delta t\left\|\Delta \mathbf{u}^{0}\right\|^{2}+\int_{0}^{T_{0}}\|\mathbf{f}\|^{2} \leq M_{0}
$$

then whenever $0<n \Delta t \leq T=\min \left(T_{*}, T_{0}\right)$, the solution to the time-discrete scheme (4)-(6) and (73) satisfies

$$
\begin{array}{r}
\sup _{0 \leq k \leq n}\left\|\nabla \mathbf{u}^{k}\right\|^{2}+\sum_{k=0}^{n}\left\|\Delta \mathbf{u}^{k}\right\|^{2} \Delta t \leq C_{3}, \\
\sum_{k=0}^{n-1}\left(\left\|\frac{\mathbf{u}^{k+1}-\mathbf{u}^{k}}{\Delta t}\right\|^{2}+\left\|\mathbf{u}^{k} \cdot \nabla \mathbf{u}^{k}\right\|^{2}\right) \Delta t \leq C_{3} .
\end{array}
$$




\section{Proof: Put}

$$
z_{n}=\left\|\nabla \mathbf{u}^{n}\right\|^{2}+\left(\nu-\varepsilon_{1}\right) \Delta t\left\|\Delta \mathbf{u}^{n}\right\|^{2}, \quad w_{n}=\varepsilon\left\|\Delta \mathbf{u}^{n}\right\|^{2}, \quad b_{n}=\left\|\mathbf{f}^{n}\right\|^{2},
$$

and note that from (73) we have that as long as $n \Delta t \leq T$,

$$
\sum_{k=0}^{n-1}\left\|\mathbf{f}_{k}\right\|^{2} \Delta t \leq \int_{0}^{T}|\mathbf{f}(t)|^{2} d t
$$

by the Cauchy-Schwarz inequality. Then by (86),

$$
z_{n+1}+w_{n} \Delta t \leq z_{n}+C \Delta t\left(b_{n}+z_{n}+z_{n}^{3}\right),
$$

where we have replaced $\max \left\{8 / \varepsilon_{1}, 32 C /\left(\varepsilon_{1} \varepsilon_{2}\right), \nu C_{\beta}\right\}$ by $C$. Summing from 0 to $n-1$ and using (90) yields

$$
z_{n}+\sum_{k=0}^{n-1} w_{k} \Delta t \leq C M_{0}+C \Delta t \sum_{k=0}^{n-1}\left(z_{k}+z_{k}^{3}\right)=: y_{n}
$$

The quantities $y_{n}$ so defined increase with $n$ and satisfy

$$
y_{n+1}-y_{n}=C \Delta t\left(z_{n}+z_{n}^{3}\right) \leq C \Delta t\left(y_{n}+y_{n}^{3}\right) .
$$

Now set $F(y)=\ln \left(y / \sqrt{1+y^{2}}\right)$ so that $F^{\prime}(y)=\left(y+y^{3}\right)^{-1}$. Then on $(0, \infty), F$ is negative, increasing and concave, and we have

$$
F\left(y_{n+1}\right)-F\left(y_{n}\right) \leq F^{\prime}\left(y_{n}\right)\left(y_{n+1}-y_{n}\right)=\frac{y_{n+1}-y_{n}}{y_{n}+y_{n}^{3}} \leq C \Delta t
$$

whence

$$
F\left(y_{n}\right) \leq F\left(y_{0}\right)+C n \Delta t=F\left(C M_{0}\right)+C n \Delta t .
$$

Choosing any $T_{*}>0$ so that $C_{*}:=F\left(C M_{0}\right)+C T_{*}<0$, we infer that as long as $n \Delta t \leq T_{*}$ we have $y_{n} \leq F^{-1}\left(C_{*}\right)$, and this together with (92) yields the stability estimate $(87)$.

Now, using (85) and elliptic regularity, we get from (87) that

$$
\sum_{k=0}^{n}\left\|\mathbf{u}^{k} \cdot \nabla \mathbf{u}^{k}\right\|^{2} \Delta t \leq C \sum_{k=0}^{n}\left\|\nabla \mathbf{u}^{k}\right\|_{L^{2}}^{2}\left\|\nabla \mathbf{u}^{k}\right\|_{H^{1}}^{2} \Delta t \leq C \sum_{k=0}^{n}\left\|\Delta \mathbf{u}^{k}\right\|^{2} \Delta t \leq C .
$$

Then the difference equation (5) yields

$$
\sum_{k=0}^{n-1}\left\|\frac{\mathbf{u}^{k+1}-\mathbf{u}^{k}}{\Delta t}\right\|^{2} \Delta t \leq C
$$

This yields (88) and finishes the proof of the Theorem. 


\section{Existence, uniqueness, convergence}

The stability estimates in Theorem 2 lead in classic fashion to a short proof of existence and uniqueness for strong solutions of the unconstrained formulation (9) of the Navier-Stokes equations. Convergence of the time-discrete scheme follows as a consequence. Regarding the constrained Navier-Stokes equations there are of course many previous works; see [Am1] for a recent comprehensive treatment. For unconstrained formulations of the Navier-Stokes equations with a variety of boundary conditions including the one considered in the present paper, Grubb and Solonnikov [GS1, GS2] lay out a general existence theory in anisotropic Sobolev spaces using a theory of pseudodifferential initial-boundaryvalue problems developed by Grubb.

Theorem 3 Let $\Omega$ be a bounded domain in $\mathbb{R}^{3}$ with boundary $\Gamma$ of class $C^{3}$, and let $\nu, M_{1}>0$. Then, there exists $T_{*}>0$ such that if $\mathbf{f} \in L^{2}\left(0, T_{0} ; L^{2}\left(\Omega, \mathbb{R}^{N}\right)\right)$ for some $T_{0}>0$ and $\mathbf{u}_{\mathrm{in}} \in H_{0}^{1}\left(\Omega, \mathbb{R}^{N}\right)$ with $\left\|\nabla \mathbf{u}_{\mathrm{in}}\right\|^{2}+\int_{0}^{T_{0}}\|\mathbf{f}\|^{2} \leq M_{1}$, then a unique strong solution of (11)-(13) exists on $[0, T], T=\min \left(T_{*}, T_{0}\right)$, that satisfies (72) and

$$
\begin{aligned}
& \mathbf{u} \in L^{2}\left(0, T ; H^{2}\left(\Omega, \mathbb{R}^{N}\right)\right) \cap H^{1}\left(0, T ; L^{2}\left(\Omega, \mathbb{R}^{N}\right)\right), \\
& \nabla p \in L^{2}\left(0, T ; L^{2}\left(\Omega, \mathbb{R}^{N}\right)\right),
\end{aligned}
$$

Moreover, $\mathbf{u} \in C\left([0, T], H^{1}\left(\Omega, \mathbb{R}^{N}\right)\right)$, and $\nabla \cdot \mathbf{u} \in C^{\infty}\left((0, T], C^{\infty}(\Omega)\right)$ is a classical solution of the heat equation with no-flux boundary conditions. The map $t \mapsto\|\nabla \cdot \mathbf{u}\|^{2}$ is smooth for $t>0$ and we have the dissipation identity

$$
\frac{d}{d t} \frac{1}{2}\|\nabla \cdot \mathbf{u}\|^{2}+\nu\|\nabla(\nabla \cdot \mathbf{u})\|^{2}=0
$$

Proof of existence: We shall give a simple proof of existence based on the finite difference scheme considered in section 4, using a classical compactness argument [Tar, Te1, LM]. In contrast to similar arguments in other sources, for example by Temam [Te1] for a time-discrete scheme with implicit differencing of pressure terms, we do not make any use of regularity theory for stationary Stokes systems.

First we smooth the initial data. Given $\mathbf{u}_{\text {in }} \in H_{0}^{1}\left(\Omega, \mathbb{R}^{N}\right)$ and $\Delta t>0$, determine $\mathbf{u}^{0}$ in $H_{0}^{1} \cap H^{2}\left(\Omega, \mathbb{R}^{N}\right)$ by solving $(I-\Delta t \Delta) \mathbf{u}^{0}=\mathbf{u}_{\text {in }}$. An energy estimate yields

$$
\left\|\nabla \mathbf{u}^{0}\right\|^{2}+\Delta t\left\|\Delta \mathbf{u}^{0}\right\|^{2} \leq\left\|\nabla \mathbf{u}_{\text {in }}\right\|\left\|\nabla \mathbf{u}^{0}\right\| \leq\left\|\nabla \mathbf{u}_{\text {in }}\right\|^{2} .
$$

Then $\left\|\Delta t \Delta \mathbf{u}^{0}\right\|^{2}=O(\Delta t)$ as $\Delta t \rightarrow 0$, so $\mathbf{u}^{0} \rightarrow \mathbf{u}_{\text {in }}$ strongly in $L^{2}$ and weakly in $H^{1}$. Note the stability constant $C_{3}$ in Theorem 2 is independent of $\Delta t$.

We define the discretized solution $\mathbf{u}^{n}$ by (4)-(6), and note

(98) $\frac{\mathbf{u}^{n+1}-\mathbf{u}^{n}}{\Delta t}+\mathcal{P}\left(\mathbf{u}^{n} \cdot \nabla \mathbf{u}^{n}-\mathbf{f}^{n}-\nu \Delta \mathbf{u}^{n}\right)=\nu \Delta\left(\mathbf{u}^{n+1}-\mathbf{u}^{n}\right)+\nu \nabla \nabla \cdot \mathbf{u}^{n}$. 
With $t_{n}=n \Delta t$, we put $\mathbf{u}_{\Delta t}\left(t_{n}\right)=\mathbf{U}_{\Delta t}\left(t_{n}\right)=\mathbf{u}_{n}$ for $n=0,1,2, \ldots$, and define $\mathbf{u}_{\Delta t}(t)$ and $\mathbf{U}_{\Delta t}(t)$ on each subinterval $\left[t_{n}, t_{n}+\Delta t\right)$ through linear interpolation and as piecewise constant respectively:

$$
\begin{aligned}
\mathbf{u}_{\Delta t}\left(t_{n}+s\right) & =\mathbf{u}^{n}+s\left(\frac{\mathbf{u}^{n+1}-\mathbf{u}^{n}}{\Delta t}\right), & s \in[0, \Delta t), \\
\mathbf{U}_{\Delta t}\left(t_{n}+s\right) & =\mathbf{u}^{n}, & s \in[0, \Delta t) .
\end{aligned}
$$

Then (98) means that whenever $t>0$ with $t \neq t_{n}$,

(101)

$\partial_{t} \mathbf{u}_{\Delta t}+\mathcal{P}\left(\mathbf{U}_{\Delta t} \cdot \nabla \mathbf{U}_{\Delta t}-\mathbf{f}_{\Delta t}-\nu \Delta \mathbf{U}_{\Delta t}\right)=\nu \Delta\left(\mathbf{U}_{\Delta t}(\cdot+\Delta t)-\mathbf{U}_{\Delta t}\right)+\nu \nabla \nabla \cdot \mathbf{U}_{\Delta t}$,

where $\mathbf{f}_{\Delta t}(t)=\mathbf{f}^{n}$ for $t \in\left[t_{n}, t_{n}+\Delta t\right)$.

We will use the simplified notation $X(Y)$ to denote a function space of the form $X\left([0, T], Y\left(\Omega, \mathbb{R}^{N}\right)\right)$, and we let $Q=\Omega \times[0, T]$ where $T=\min \left(T_{0}, T_{*}\right)$ with $T_{*}$ as given by Theorem 2 . The estimates in Theorem 2 say that $\mathbf{u}_{\Delta t}$ is bounded in the Hilbert space

$$
V_{0}:=L^{2}\left(H^{2} \cap H_{0}^{1}\right) \cap H^{1}\left(L^{2}\right),
$$

and also that $\mathbf{U}_{\Delta t}$ is bounded in $L^{2}\left(H^{2}\right)$, uniformly for $\Delta t>0$. Moreover, estimate (87) says $\mathbf{u}_{\Delta t}$ is bounded in $C\left(H^{1}\right)$. This is also a consequence of the embedding $V_{0} \hookrightarrow C\left(H^{1}\right)$, see [Tar, p. 42] or [Ev, p. 288].

Along some subsequence $\Delta t_{j} \rightarrow 0$, then, we have that $\mathbf{u}_{\Delta t}$ converges weakly in $V_{0}$ to some $\mathbf{u} \in V_{0}$, and $\mathbf{U}_{\Delta t}$ and $\mathbf{U}_{\Delta t}(\cdot+\Delta t)$ converge weakly in $L^{2}\left(H^{2}\right)$ to some $\mathbf{U}_{1}$ and $\mathbf{U}_{2}$ respectively. Since clearly $V_{0} \hookrightarrow H^{1}(Q)$, and since the embedding $H^{1}(Q) \hookrightarrow L^{2}(Q)$ is compact, we have that $\mathbf{u}_{\Delta t} \rightarrow \mathbf{u}$ strongly in $L^{2}(Q)$. Note that by estimate (88),

$$
\left\|\mathbf{u}_{\Delta t}-\mathbf{U}_{\Delta t}\right\|_{L^{2}(Q)}^{2} \leq\left\|\mathbf{U}_{\Delta t}(\cdot+\Delta t)-\mathbf{U}_{\Delta t}\right\|_{L^{2}(Q)}^{2}=\sum_{k=0}^{n-1}\left\|\mathbf{u}^{n+1}-\mathbf{u}^{n}\right\|^{2} \Delta t \leq C \Delta t^{2} .
$$

Therefore $\mathbf{U}_{\Delta t}(\cdot+\Delta t)$ and $\mathbf{U}_{\Delta t}$ converge to $\mathbf{u}$ strongly in $L^{2}(Q)$ also, so $\mathbf{U}_{1}=$ $\mathbf{U}_{2}=\mathbf{u}$.

We want to show $\mathbf{u}$ is a strong solution of (11) by passing to the limit in (101). From the definition of $\mathbf{f}^{n}$ in (73) and estimate (90), it is a standard result which can be proved by using the density of $C(Q)$ in $L^{2}(Q)$ that

$$
\left\|\mathbf{f}-\mathbf{f}_{\Delta t}\right\|_{L^{2}(Q)}^{2} \rightarrow 0 \quad \text { as } \Delta t \rightarrow 0 .
$$

We are now justified in passing to the limit weakly in $L^{2}(Q)$ in all terms in (101) except the nonlinear term, which (therefore) converges weakly to some $\mathbf{w} \in L^{2}(Q)$. But since $\nabla \mathbf{U}_{\Delta t}$ converges to $\nabla \mathbf{u}$ weakly and $\mathbf{U}_{\Delta t}$ to $\mathbf{u}$ strongly in $L^{2}(Q)$, we can conclude $\mathbf{U}_{\Delta t} \cdot \nabla \mathbf{U}_{\Delta t}$ converges to $\mathbf{u} \cdot \nabla \mathbf{u}$ in the sense of distributions on $Q$. So $\mathbf{w}=\mathbf{u} \cdot \nabla \mathbf{u}$, and upon taking limits in (101) it follows that

$$
\partial_{t} \mathbf{u}+\mathcal{P}(\mathbf{u} \cdot \nabla \mathbf{u}-\mathbf{f}-\nu \Delta \mathbf{u})=\nu \nabla \nabla \cdot \mathbf{u}
$$


That is, $\mathbf{u}$ is indeed a strong solution of (11). That $\mathbf{u}(0)=\mathbf{u}_{\text {in }}$ is a consequence of the continuity of the map $\mathbf{u} \rightarrow \mathbf{u}(0)$ from $V_{0}$ through $C\left(H^{1}\right)$ to $H^{1}\left(\Omega, \mathbb{R}^{N}\right)$.

It remains to study $\nabla \cdot \mathbf{u}$. Dot (105) with $\nabla \phi, \phi \in H^{1}(\Omega)$. We get

$$
\int_{\Omega} \partial_{t} \mathbf{u} \cdot \nabla \phi=\nu \int_{\Omega} \nabla(\nabla \cdot \mathbf{u}) \cdot \nabla \phi .
$$

This says that $w=\nabla \cdot \mathbf{u}$ is a weak solution of the heat equation with Neumann boundary conditions:

$$
\partial_{t} w=\nu \Delta w \quad \text { in } \Omega, \quad \mathbf{n} \cdot \nabla w=0 \quad \text { on } \Gamma .
$$

Indeed, the operator $A:=\nu \Delta$ defined on $L^{2}(\Omega)$ with domain

$$
D(A)=\left\{w \in H^{2}(\Omega) \mid \mathbf{n} \cdot \nabla w=0 \text { on } \Gamma\right\}
$$

is self-adjoint and non-positive, so generates an analytic semigroup. For any $\phi \in D(A)$ we have that $t \mapsto\langle w(t), \phi\rangle=-\langle u(t), \nabla \phi\rangle$ is absolutely continuous, and using (106) we get $(d / d t)\langle w(t), \phi\rangle=\langle w(t), A \phi\rangle$ for a. e. $t$. By Ball's characterization of weak solutions of abstract evolution equations [Ba], $w(t)=e^{A t} w(0)$ for all $t \in[0, T]$. It follows $w \in C\left([0, T], L^{2}(\Omega)\right)$, and $w(t) \in D\left(A^{m}\right)$ for every $m>0\left[\mathrm{~Pa}\right.$, theorem 6.13]. Since $A^{m} w(t)=e^{A(t-\tau)} A^{m} w(\tau)$ if $0<\tau<t$ we infer that for $0<t \leq T, w(t)$ is analytic in $t$ with values in $D\left(A^{m}\right)$. Using interior estimates for elliptic equations, we find $w \in C^{\infty}\left((0, T], C^{\infty}(\Omega)\right)$ as desired. The dissipation identity follows by dotting with $w$.

This finishes the proof of existence.

Proof of uniqueness: Suppose $\mathbf{u}_{1}$ and $\mathbf{u}_{2}$ are both solutions of (11)-(13) and (72) belonging to $V_{0}$. Put $\mathbf{u}=\mathbf{u}_{1}-\mathbf{u}_{2}$ and $\nabla p_{\mathrm{S}}=(I-\mathcal{P})(\Delta-\nabla \nabla \cdot) \mathbf{u}$. Then $\mathbf{u}(0)=0$ and

$$
\partial_{t} \mathbf{u}+\mathcal{P}\left(\mathbf{u}_{1} \cdot \nabla \mathbf{u}+\mathbf{u} \cdot \nabla \mathbf{u}_{2}\right)=\nu \Delta \mathbf{u}-\nu \nabla p_{\mathrm{S}} .
$$

Dot with $-\Delta \mathbf{u}$ and use Theorem 1 to get

$$
\left\langle\nu \Delta \mathbf{u}-\nu \nabla p_{\mathrm{S}},-\Delta \mathbf{u}\right\rangle \leq-\frac{\nu}{2}\|\Delta \mathbf{u}\|^{2}+\frac{\nu}{2}\left\|\nabla p_{\mathrm{S}}\right\|^{2} \leq-\frac{\nu \beta}{2}\|\Delta \mathbf{u}\|^{2}+C\|\nabla \mathbf{u}\|^{2} .
$$

with $\beta=\frac{1}{2}-\varepsilon>0$. Next, use the Cauchy-Schwarz inequality for the nonlinear terms, estimating them as follows in a manner similar to (82)-(84), using that $\mathbf{u}_{1}$ and $\mathbf{u}_{2}$ are a priori bounded in $H^{1}$ norm:

$$
\left\|\mathbf{u}_{1} \cdot \nabla \mathbf{u}\right\|\|\Delta \mathbf{u}\| \leq C\left\|\nabla \mathbf{u}_{1}\right\|\|\nabla \mathbf{u}\|^{1 / 2}\|\Delta \mathbf{u}\|^{3 / 2} \leq \varepsilon\|\Delta \mathbf{u}\|^{2}+C\|\nabla \mathbf{u}\|^{2},
$$

$$
\left\|\mathbf{u} \cdot \nabla \mathbf{u}_{2}\right\|\|\Delta \mathbf{u}\| \leq C\|\nabla \mathbf{u}\|\left\|\nabla \mathbf{u}_{2}\right\|_{H^{1}}\|\Delta \mathbf{u}\| \leq \varepsilon\|\Delta \mathbf{u}\|^{2}+C\left\|\Delta \mathbf{u}_{2}\right\|^{2}\|\nabla \mathbf{u}\|^{2} .
$$

Lastly, since $\mathbf{u} \in V_{0}$ we infer that $\left\langle\partial_{t} \mathbf{u},-\Delta \mathbf{u}\right\rangle \in L^{1}(0, T)$ and $t \mapsto\|\nabla \mathbf{u}\|^{2}$ is absolutely continuous with

$$
\left\langle\partial_{t} \mathbf{u},-\Delta \mathbf{u}\right\rangle=\frac{1}{2} \frac{d}{d t}\|\nabla \mathbf{u}\|^{2}
$$


This can be shown by using the density of smooth functions in $V_{0}$; see [Ev, p. 287] for a detailed proof of a similar result.

Through this quite standard-style approach, we get

$$
\frac{d}{d t}\|\nabla \mathbf{u}\|^{2}+\alpha\|\Delta \mathbf{u}\|^{2} \leq C\left(1+\left\|\Delta \mathbf{u}_{2}\right\|^{2}\right)\|\nabla \mathbf{u}\|^{2}
$$

for some positive constants $\alpha$ and $C$. Because $\left\|\Delta \mathbf{u}_{2}\right\|^{2} \in L^{1}(0, T)$, by Gronwall's inequality we get $\|\nabla \mathbf{u}\| \equiv 0$. This proves the uniqueness.

Since the interval of existence $[0, T]$ depends only upon $M_{1}$, in standard fashion we may extend the unique strong solution to a maximal interval of time, and infer that the approximations considered above converge to this solution up to the maximal time.

Corollary 1 Given the assumptions of Theorem 3, system (11)-(13) with (72) admits a unique strong solution $\mathbf{u}$ on a maximal interval $\left[0, T_{\max }\right)$ with the property that if $T_{\max }<T_{0}$ then

$$
\|\mathbf{u}(t)\|_{H^{1}} \rightarrow \infty \quad \text { as } t \rightarrow T_{\max }
$$

For every $\hat{T} \in\left[0, T_{\max }\right)$, the approximations $\mathbf{u}_{\Delta t}$ constructed in (99) converge to $\mathbf{u}$ weakly in

$$
L^{2}\left([0, \hat{T}], H^{2} \cap H_{0}^{1}\left(\Omega, \mathbb{R}^{N}\right)\right) \cap H^{1}\left([0, \hat{T}], L^{2}\left(\Omega, \mathbb{R}^{N}\right)\right)
$$

and strongly in $L^{2}\left([0, \hat{T}] \times \Omega, \mathbb{R}^{N}\right)$.

\section{$6 \quad$ Finite element methods without compatibil- ity conditions for velocity and pressure}

The simplicity of the stability proof for the time-discrete scheme in section 4 allows us to easily establish unconditional stability locally in time, and convergence up to the maximal time of existence for the strong solution, for corresponding fully discrete finite-element methods that use $C^{1}$ elements for the velocity field and $C^{0}$ elements for pressure.

We suppose that for some sequence of positive values of $h$ approaching zero, $X_{h} \subset H^{2} \cap H_{0}^{1}\left(\Omega, \mathbb{R}^{N}\right)$ is a finite-dimensional space containing the approximate velocity field, and suppose $Y_{h} \subset H^{1}(\Omega) / \mathbb{R}$ is a finite-dimensional space containing approximate pressures. We assume these spaces have the approximation property that

$$
\begin{array}{r}
\forall \mathbf{v} \in H^{2} \cap H_{0}^{1}\left(\Omega, \mathbb{R}^{N}\right) \forall h \exists \mathbf{v}_{h} \in X_{h}, \quad\left\|\Delta\left(\mathbf{v}-\mathbf{v}_{h}\right)\right\| \rightarrow 0 \quad \text { as } h \rightarrow 0, \\
\forall \phi \in H^{1}(\Omega) / \mathbb{R} \forall h \exists \phi_{h} \in Y_{h}, \quad\left\|\nabla\left(\phi-\phi_{h}\right)\right\| \rightarrow 0 \quad \text { as } h \rightarrow 0 .
\end{array}
$$

As we have emphasized in the introduction to this paper, we impose no compatibility condition between the spaces $X_{h}$ and $Y_{h}$ in order to ensure an inf-sup condition. 
The finite element scheme that we consider was derived in [JL] and is equivalent to a space discretization of the scheme in (4)-(6). Given the approximate velocity $\mathbf{u}_{h}^{n}$ at the $n$-th time step, we determine $p_{h}^{n} \in Y_{h}$ and $\mathbf{u}_{h}^{n+1} \in X_{h}$ by requiring

$$
\begin{array}{r}
\left\langle\frac{\nabla \mathbf{u}_{h}^{n+1}-\nabla \mathbf{u}_{h}^{n}}{\Delta t}, \nabla \mathbf{v}_{h}\right\rangle+\left\langle\nu \Delta \mathbf{u}_{h}^{n+1}, \Delta \mathbf{v}_{h}\right\rangle=\left\langle\nabla p_{h}^{n}+\mathbf{u}_{h}^{n} \cdot \nabla \mathbf{u}_{h}^{n}-\mathbf{f}^{n}, \Delta \mathbf{v}_{h}\right\rangle \\
\forall \mathbf{v}_{h} \in X_{h} .
\end{array}
$$

We remark that in general, practical finite element methods usually use spaces defined on domains that approximate the given $\Omega$. For simplicity here we suppose $\Omega$ can be kept fixed, such that finite-element spaces $X_{h}$ and $Y_{h}$ can be found as described with $C^{1}$ elements for velocity and $C^{0}$ elements for pressure. In principle this should be possible whenever $\Omega$ has a piecewise polynomial $C^{3}$ boundary.

Stability. We are to show the scheme in (118)-(119) is unconditionally stable. First, we take $\phi_{h}=p_{h}^{n}$ in (118). Due to the fact that

$$
\left\langle\mathcal{P}(\Delta-\nabla \nabla \cdot) \mathbf{u}_{h}^{n}, \nabla p_{h}^{n}\right\rangle=0,
$$

we directly deduce from the Cauchy-Schwarz inequality that

$$
\left\|\nabla p_{h}^{n}\right\| \leq\left\|\nu \nabla p_{\mathrm{S}}\left(\mathbf{u}_{h}^{n}\right)\right\|+\left\|\mathbf{u}_{h}^{n} \cdot \nabla \mathbf{u}_{h}^{n}-\mathbf{f}^{n}\right\|
$$

where

$$
\nabla p_{\mathrm{S}}\left(\mathbf{u}_{h}^{n}\right)=(\Delta \mathcal{P}-\mathcal{P} \Delta) \mathbf{u}_{h}^{n}=(I-\mathcal{P})(\Delta-\nabla \nabla \cdot) \mathbf{u}_{h}^{n}
$$

is the Stokes pressure associated with $\mathbf{u}_{h}^{n}$. (Note $\nabla p_{\mathrm{S}}\left(\mathbf{u}_{h}^{n}\right)$ need not lie in the space $Y_{h}$.) Now, taking $\mathbf{v}_{h}=\mathbf{u}_{h}^{n+1}$ in (119) and arguing just as in (76), we obtain an exact analog of (77), namely

$$
\begin{aligned}
& \frac{1}{\Delta t}\left(\left\|\nabla \mathbf{u}_{h}^{n+1}\right\|^{2}-\left\|\nabla \mathbf{u}_{h}^{n}\right\|^{2}\right)+\left(\nu-\varepsilon_{1}\right)\left\|\Delta \mathbf{u}_{h}^{n+1}\right\|^{2} \\
& \leq \frac{8}{\varepsilon_{1}}\left(\left\|\mathbf{f}^{n}\right\|^{2}+\left\|\mathbf{u}_{h}^{n} \cdot \nabla \mathbf{u}_{h}^{n}\right\|^{2}\right)+\nu\left\|\nabla p_{\mathrm{S}}\left(\mathbf{u}_{h}^{n}\right)\right\|^{2} .
\end{aligned}
$$

Proceeding now exactly as in section 4 leads to the following unconditional stability result.

Theorem 4 Let $\Omega$ be a bounded domain in $\mathbb{R}^{N}(N=2$ or 3$)$ with $C^{3}$ boundary, and let $\nu, M_{0}>0$. Then there exist positive constants $T_{*}$ and $C_{4}$ with the following property. Suppose $X_{h} \subset H^{2} \cap H_{0}^{1}\left(\Omega, \mathbb{R}^{N}\right), Y_{h} \subset H^{1}(\Omega) / \mathbb{R}$ satisfy (116)-(117). Assume $\mathbf{f} \in L^{2}\left(0, T_{0} ; L^{2}\left(\Omega, \mathbb{R}^{N}\right)\right)$ for some $T_{0}>0, \mathbf{u}_{h}^{0} \in X_{h}$ and

$$
\left\|\nabla \mathbf{u}_{h}^{0}\right\|^{2}+\nu \Delta t\left\|\Delta \mathbf{u}_{h}^{0}\right\|^{2}+\int_{0}^{T_{0}}\|\mathbf{f}\|^{2} \leq M_{0} .
$$


Then whenever $0<n \Delta t \leq T=\min \left(T_{*}, T_{0}\right)$, the solution to the finite-element scheme (118)-(119) satisfies

$$
\begin{array}{r}
\sup _{0 \leq k \leq n}\left\|\nabla \mathbf{u}_{h}^{k}\right\|^{2}+\sum_{k=0}^{n}\left\|\Delta \mathbf{u}_{h}^{k}\right\|^{2} \Delta t \leq C_{4}, \\
\sum_{k=0}^{n-1}\left(\left\|\frac{\mathbf{u}_{h}^{k+1}-\mathbf{u}_{h}^{k}}{\Delta t}\right\|^{2}+\left\|\mathbf{u}_{h}^{k} \cdot \nabla \mathbf{u}_{h}^{k}\right\|^{2}\right) \Delta t \leq C_{4} .
\end{array}
$$

Convergence. We prove the convergence of the finite-element scheme described above by taking $h \rightarrow 0$ to obtain the solution of the time-discrete scheme studied in section 4 , then $\Delta t \rightarrow 0$ as before. Because of the uniqueness of the solution of the time-discrete scheme and of the strong solution of the PDE, it suffices to prove convergence for some subsequence of any given sequence of values of $h$ tending toward 0 . The bounds obtained in Theorem 4 make this rather straightforward.

Fix $\Delta t>0$. The bounds in Theorem 4 and in (120) imply that for all positive integers $n<T_{*} / \Delta t$, the $\mathbf{u}_{h}^{n}$ are bounded in $H^{2} \cap H_{0}^{1}\left(\Omega, \mathbb{R}^{N}\right)$ and the $\nabla p_{h}^{n}$ are bounded in $L^{2}\left(\Omega, \mathbb{R}^{N}\right)$ uniformly in $h$. So from any sequence of $h$ approaching zero, we may extract a subsequence along which we have weak limits

$$
\mathbf{u}_{h}^{n} \rightarrow \mathbf{u}^{n} \text { in } H^{2}\left(\Omega, \mathbb{R}^{N}\right), \quad \nabla p_{h}^{n} \rightarrow \nabla p^{n}, \mathbf{u}_{h}^{n} \cdot \nabla \mathbf{u}_{h}^{n} \rightarrow \mathbf{w}^{n} \text { in } L^{2}\left(\Omega, \mathbb{R}^{N}\right)
$$

for all $n$. Then $\mathbf{u}_{h}^{n} \rightarrow \mathbf{u}^{n}$ strongly in $H_{0}^{1}\left(\Omega, \mathbb{R}^{N}\right)$ and so $\mathbf{w}^{n}=\mathbf{u}^{n} \cdot \nabla \mathbf{u}^{n}$ since the nonlinear term converges strongly in $L^{1}$.

Now, for any $\mathbf{v} \in H^{2} \cap H_{0}^{1}\left(\Omega, \mathbb{R}^{N}\right)$ and $\phi \in H^{1}(\Omega)$, by assumption there exist $\mathbf{v}_{h} \in X_{h}, \phi_{h} \in H^{1}(\Omega)$ such that $\mathbf{v}_{h} \rightarrow \mathbf{v}$ strongly in $H^{2}\left(\Omega, \mathbb{R}^{N}\right)$ and $\nabla \phi_{h} \rightarrow \nabla \phi$ strongly in $L^{2}\left(\Omega, \mathbb{R}^{N}\right)$. Applying these convergence properties in (118)-(119) yields that the weak limits in (125) satisfy

$$
\begin{aligned}
& \left\langle\nabla p^{n}+\nu \nabla \nabla \cdot \mathbf{u}^{n}-\nu \Delta \mathbf{u}^{n}+\mathbf{u}^{n} \cdot \nabla \mathbf{u}^{n}-\mathbf{f}^{n}, \nabla \phi\right\rangle=0 \\
& \left\langle\frac{\mathbf{u}^{n+1}-\mathbf{u}^{n}}{\Delta t}-\nu \Delta \mathbf{u}^{n+1}+\nabla p^{n}+\mathbf{u}^{n} \cdot \nabla \mathbf{u}^{n}-\mathbf{f}^{n}, \Delta \mathbf{v}\right\rangle=0 .
\end{aligned}
$$

But this means exactly that $\mathbf{u}^{n}$ satisfies (4)-(6). So in the limit $h \rightarrow 0$ we obtain the solution of the time-discrete scheme studied in section 4 . Then the limit $\Delta t \rightarrow 0$ yields the unique strong solution on a maximal time interval as established in section 5 .

\section{Non-homogeneous side conditions}

We consider the Navier-Stokes equations with non-homogeneous boundary conditions and extended to include a divergence constraint (such as may be used to model a distributed source or sink of fluid at constant density, with an ad- 
justment of the body force $\mathbf{f}$ to account for bulk viscosity):

$$
\begin{aligned}
\partial_{t} \mathbf{u}+\mathbf{u} \cdot \nabla \mathbf{u}+\nabla p-\nu \Delta \mathbf{u}=\mathbf{f} & \text { in } \Omega \text { for } t>0, \\
\nabla \cdot \mathbf{u}=h & \text { in } \Omega \text { for } t \geq 0, \\
\mathbf{u}=\mathbf{g} & \text { on } \Gamma \text { for } t \geq 0, \\
\mathbf{u}=\mathbf{u}_{\text {in }} & \text { in } \Omega \text { for } t=0 .
\end{aligned}
$$

Our aim in this section is to prove stability, and convergence to strong solutions, for a time-discretization of an appropriate unconstrained formulation of this problem. In a subsequent work we will study error estimates for fully discrete finite element methods, with greater regularity assumptions on the data.

\subsection{An unconstrained formulation}

We can write an unconstrained formulation for (128) similar to (9), but including another gradient term to account for the inhomogeneous side conditions:

$$
\partial_{t} \mathbf{u}+\mathcal{P}(\mathbf{u} \cdot \nabla \mathbf{u}-\mathbf{f}-\nu \Delta \mathbf{u})+\nabla p_{g h}=\nu \nabla(\nabla \cdot \mathbf{u}) .
$$

We obtain an equation that determines the inhomogeneous pressure $p_{g h}$ by imposing the requirement that the divergence residual $w:=\nabla \cdot \mathbf{u}-h$ should be a weak solution of the heat equation with no-flux boundary conditions:

$$
\partial_{t} w=\nu \Delta w \text { in } \Omega, \quad \mathbf{n} \cdot \nabla w=0 \text { on } \Gamma,
$$

and with initial condition $w=\nabla \cdot \mathbf{u}_{\text {in }}-\left.h\right|_{t=0}$. Then the divergence constraint will be enforced through exponential diffusive decay as before. (See (174) below.) By formal integration by parts using the side conditions, we find that an appropriate weak formulation of (133) is to require

$$
\left\langle\partial_{t} \mathbf{u}, \nabla \phi\right\rangle-\left\langle\partial_{t}(\mathbf{n} \cdot \mathbf{g}), \phi\right\rangle_{\Gamma}+\left\langle\partial_{t} h, \phi\right\rangle=\langle\nu \nabla(\nabla \cdot \mathbf{u}-h), \nabla \phi\rangle
$$

for all $\phi \in H^{1}(\Omega)$. To obtain this from (132), we should require

$$
\left\langle\nabla p_{g h}, \nabla \phi\right\rangle=-\left\langle\partial_{t}(\mathbf{n} \cdot \mathbf{g}), \phi\right\rangle_{\Gamma}+\left\langle\partial_{t} h, \phi\right\rangle+\langle\nu \nabla h, \nabla \phi\rangle \quad \forall \phi \in H^{1}(\Omega) .
$$

See Lemma 5 below regarding the existence of $p_{g h}$ satisfying (135) for data $\mathbf{g}$ and $h$ in an appropriate class.

As with (14), by using Lemma 1 and the definition $\nabla p_{\mathrm{S}}=(\Delta \mathcal{P}-\mathcal{P} \Delta) \mathbf{u}$, we find that (132) is equivalent to

$$
\partial_{t} \mathbf{u}+\mathcal{P}(\mathbf{u} \cdot \nabla \mathbf{u}-\mathbf{f})+\nabla p_{g h}+\nu \nabla p_{\mathrm{S}}=\nu \Delta \mathbf{u} .
$$

Our unconstrained formulation of (128)-(131) then takes the form (135)-(136) together with (130)-(131). 


\subsection{Regularity assumptions}

We assume $\Omega \subset \mathbb{R}^{N}$ ( $N=2$ or 3 ) is a connected bounded domain with boundary $\Gamma$ of class $C^{3}$ as before. Let

$$
\begin{aligned}
V(0, T) & :=L^{2}\left(0, T ; H^{2}(\Omega)\right) \cap H^{1}\left(0, T ; L^{2}(\Omega)\right), \\
W(0, T) & :=L^{2}\left(0, T ; H^{1}(\Omega)\right) \cap H^{1}\left(0, T ; H^{1}(\Omega)^{\prime}\right) .
\end{aligned}
$$

Here $\left(H^{1}\right)^{\prime}$ is the space dual to $H^{1}$.

Our theory of convergence to strong solutions comes in two similar flavors, depending on the regularity assumed on the data. The two flavors correspond to solutions having either the regularity

$$
\mathbf{u} \in V_{\operatorname{div}}(0, T):=V(0, T)^{N} \cap\{\mathbf{u} \mid \nabla \cdot \mathbf{u} \in V(0, T)\},
$$

or the somewhat weaker regularity

$$
\mathbf{u} \in W_{\operatorname{div}}(0, T):=V(0, T)^{N} \cap\{\mathbf{u} \mid \nabla \cdot \mathbf{u} \in W(0, T)\},
$$

for some $T>0$. In the first case, $\nabla \cdot \mathbf{u}$ is more regular $(\nabla \cdot \mathbf{u}=0$ is usual), but we need to assume $\nabla \cdot \mathbf{u}_{\text {in }} \in H^{1}(\Omega)$ due to the embedding $V(0, T) \hookrightarrow$ $C\left([0, T], H^{1}(\Omega)\right)$. The condition (140) means that $\mathbf{u} \in V(0, T)^{N}$ and $\nabla \cdot \mathbf{u}$ has vector-valued distributional derivative $\partial_{t}(\nabla \cdot \mathbf{u})$ in $L^{2}\left(0, T ; H^{1}(\Omega)^{\prime}\right)$, the dual of $L^{2}\left(0, T ; H^{1}(\Omega)\right)$.

It will be a consequence of our theory that an arbitrary pair $(\mathbf{u}, p)$ with

$$
\mathbf{u} \in V_{\mathrm{div}}(0, T) \text { or } W_{\mathrm{div}}(0, T), \quad p \in L^{2}\left(0, T ; H^{1}(\Omega) / \mathbb{R}\right)
$$

is a strong solution of the constrained system (128)-(131) for appropriate data. For the linear problem without convection term, this yields an isomorphism between the solution space from (141) for the constrained Stokes problem and the corresponding space of data that satisfy the requirements below together with the extra compatibility condition

$$
\nabla \cdot \mathbf{u}_{\text {in }}=h \quad \text { in } \Omega \text { for } t=0 .
$$

Corresponding to the regularity in (140), our precise assumptions on the data are that for some $T>0$ we have

$$
\begin{aligned}
\mathbf{f} \in H_{f}: & : L^{2}\left(0, T ; L^{2}\left(\Omega, \mathbb{R}^{N}\right)\right), \\
\mathbf{g} \in H_{g}:=H^{3 / 4}\left(0, T ; L^{2}\left(\Gamma, \mathbb{R}^{N}\right)\right) \cap L^{2}\left(0, T ; H^{3 / 2}\left(\Gamma, \mathbb{R}^{N}\right)\right) & \\
& \quad \cap\left\{\mathbf{g} \mid \partial_{t}(\mathbf{n} \cdot \mathbf{g}) \in L^{2}\left(0, T ; H^{-1 / 2}(\Gamma)\right)\right\}, \\
h \in H_{h}:=W(0, T), & \\
\mathbf{u}_{\text {in }} \in H_{\text {in }}: & =H^{1}\left(\Omega, \mathbb{R}^{N}\right) .
\end{aligned}
$$

We also impose the compatibility conditions

$$
\begin{aligned}
\int_{\Gamma} \mathbf{n} \cdot \mathbf{g}=\int_{\Omega} h \quad \text { for } t>0 \\
\mathbf{g}=\mathbf{u}_{\text {in }} \quad \text { on } \Gamma \text { for } t=0 .
\end{aligned}
$$


Corresponding to the regularity in (139), we will assume additionally that $h \in$ $V(0, T)$ and $\nabla \cdot \mathbf{u}_{\text {in }} \in H^{1}(\Omega)$.

With these assumptions, we get the existence of $p_{g h}$ as follows.

Lemma 5 Assume (144), (145) and (147). Then there exists $p_{g h} \in L^{2}\left(H^{1}(\Omega)\right)$ with zero mean, satisfying (135) and

$$
\left\|p_{g h}\right\|_{L^{2}\left(H^{1}(\Omega)\right)} \leq C\left(\left\|\partial_{t}(\mathbf{n} \cdot \mathbf{g})\right\|_{L^{2}\left(H^{-1 / 2}(\Gamma)\right)}+\|h\|_{W(0, T)}\right) .
$$

Proof: One applies the Lax-Milgram lemma for a.e. $t$ to (135) in the space of functions in $H^{1}(\Omega)$ with zero average. We omit the standard details.

To explain the regularity of $\mathbf{g}$, and eventually reduce the analysis to a problem with homogeneous boundary conditions, we make the following point. From the theory of Lions and Magenes [LM] (see Theorems 2.3 and 4.3 in vol. II), taking the trace on the parabolic boundary of $\Omega \times(0, T)$, defined for smooth enough functions by $u \mapsto\left(u(0, \cdot),\left.u\right|_{\Gamma}\right)$, extends to yield a bounded map

$$
\begin{gathered}
V(0, T) \rightarrow H^{1}(\Omega) \times\left(H^{3 / 4}\left(0, T ; L^{2}(\Gamma)\right) \cap L^{2}\left(0, T ; H^{3 / 2}(\Gamma)\right)\right) \\
\cap\{(u, g) \mid u=g \text { on } \Gamma \text { for } t=0\},
\end{gathered}
$$

and this map admits a bounded right inverse. By consequence, given $\left(\mathbf{u}_{\text {in }}, \mathbf{g}\right)$ satisfying our assumptions above, there exists $\tilde{\mathbf{u}}$ such that

$$
\tilde{\mathbf{u}} \in V(0, T)^{N}, \quad \tilde{\mathbf{u}}(0)=\mathbf{u}_{\mathrm{in}},\left.\quad \tilde{\mathbf{u}}\right|_{\Gamma}=\mathbf{g},
$$

and the norm of $\tilde{\mathbf{u}}$ in $V(0, T)^{N}$ is bounded in terms of the norm of $\left(\mathbf{u}_{\text {in }}, \mathbf{g}\right)$ in $H_{i n} \times H_{g}$. One can regard $\tilde{\mathbf{u}}$ as given data, instead of the pair $\left(\mathbf{u}_{\mathrm{in}}, \mathbf{g}\right)$.

To relate the regularity in (140) with (144), we note the following.

Lemma $6 \quad W_{\text {div }}(0, T)=V(0, T)^{N} \cap\left\{\mathbf{u} \mid \partial_{t}\left(\left.\mathbf{n} \cdot \mathbf{u}\right|_{\Gamma}\right) \in L^{2}\left(0, T ; H^{-1 / 2}(\Gamma)\right)\right\}$.

Proof: Let $\mathbf{u} \in V(0, t)^{N}$. Given any $\phi \in C_{0}^{\infty}\left((0, T), H^{1}(\Omega)\right)$ we have

$$
\int_{0}^{T}\left\langle\nabla \cdot \mathbf{u}, \partial_{t} \phi\right\rangle d t=\int_{0}^{T}\left\langle\mathbf{n} \cdot \mathbf{u}, \partial_{t} \phi\right\rangle_{\Gamma} d t+\int_{0}^{T}\left\langle\partial_{t} \mathbf{u}, \nabla \phi\right\rangle d t .
$$

The last term is bounded by a constant times the norm of $\phi$ in $L^{2}\left(0, T ; H^{1}(\Omega)\right)$. Using that the trace map $H^{1}(\Omega) \rightarrow H^{1 / 2}(\Gamma)$ is onto with bounded right inverse, we get the left-hand side bounded by the same estimate if and only if the boundary term is bounded by the norm of $\left.\phi\right|_{\Gamma}$ in $L^{2}\left(0, T ; H^{1 / 2}(\Gamma)\right)$. This means $\partial_{t}(\nabla \cdot \mathbf{u}) \in L^{2}\left(0, T ; H^{1}(\Omega)^{\prime}\right)$ if and only if $\partial_{t}\left(\left.\mathbf{n} \cdot \mathbf{u}\right|_{\Gamma}\right) \in L^{2}\left(0, T ; H^{-1 / 2}(\Gamma)\right)$.

At this point we can give our "converse existence" result.

Proposition 2 Suppose that $(\mathbf{u}, p)$ is an arbitrary pair that satisfies (141), and let $\mathbf{f}, \mathbf{g}, h$ and $\mathbf{u}_{\mathrm{in}}$ be computed from (128)-(131). Then the conditions (143)(148) all hold, and $\mathbf{u}$ satisfies (135)-(136). Additionally, if $\mathbf{u} \in V_{\mathrm{div}}(0, T)$ then $h \in V(0, T)$ and $\nabla \cdot \mathbf{u}_{\text {in }} \in H^{1}(\Omega)$. 
Proof: One easily checks (143)-(148) using (85) and (150) and Lemma 6. Additionally, if $\mathbf{u} \in V_{\mathrm{div}}(0, T)$, clearly $h \in V(0, T)$ and $\nabla \cdot \mathbf{u}_{\text {in }} \in H^{1}(\Omega)$. Furthermore, for any $\phi \in C_{0}^{\infty}\left((0, T), H^{1}(\Omega)\right)$, from (152) we find

$$
-\int_{0}^{T}\left\langle\partial_{t} h, \phi\right\rangle d t=-\int_{0}^{T}\left\langle\partial_{t}(\mathbf{n} \cdot \mathbf{g}), \phi\right\rangle_{\Gamma} d t+\int_{0}^{T}\left\langle\partial_{t} \mathbf{u}, \nabla \phi\right\rangle d t
$$

Hence (134) holds in $L^{1}(0, T)$. If we define $\nabla p_{\mathrm{S}}=(\Delta \mathcal{P}-\mathcal{P} \Delta) \mathbf{u}$ and $\nabla p_{\mathrm{E}}=$ $(\mathcal{P}-I)(\mathbf{u} \cdot \nabla \mathbf{u}-\mathbf{f})$, and set $p_{g h}=p-p_{\mathrm{E}}-\nu p_{\mathrm{S}}$, it follows that (136) and (135) hold by combining (128) with Lemma 1 and (134).

We remark that most of the literature on nonhomogeneous Navier-Stokes problems [La, Sol, Gr, GS1, GS2] treats the constrained case with $h=0$ in $\Omega$ and imposes the condition $\mathbf{n} \cdot \mathbf{g}=0$ on $\Gamma$. One treatment with $h=0$ but imposing only $\int_{\Gamma} \mathbf{n} \cdot \mathbf{g}=0$ is that of Fursikov et al. $[\mathrm{Fu}]$, who study the problem in a scale of spaces that in one case exactly corresponds to what we consider here but with zero divergence constraint. Amann recently studied very weak solutions without imposing $\mathbf{n} \cdot \mathbf{g}=0$ on $\Gamma$, but only in spaces of very low regularity that exclude the present case [Am2].

\subsection{Discretization scheme}

We consider the following time-discrete scheme: Given $\mathbf{u}^{0}$, find $\mathbf{u}^{n+1}(n \geq 0)$ such that

$$
\begin{array}{rr}
\frac{\mathbf{u}^{n+1}-\mathbf{u}^{n}}{\Delta t}+\mathcal{P}\left(\mathbf{u}^{n} \cdot \nabla \mathbf{u}^{n}-\mathbf{f}^{n}\right)+\nu \nabla p_{\mathrm{S}}^{n}+\nabla p_{g h}{ }^{n}=\nu \Delta \mathbf{u}^{n+1} & \text { in } \Omega, \\
\mathbf{u}^{n+1}=\mathbf{g}^{n+1} & \text { on } \Gamma,
\end{array}
$$

where $\nabla p_{\mathrm{S}}^{n}=(\Delta \mathcal{P}-\mathcal{P} \Delta) \mathbf{u}^{n}$, and $p_{g h}{ }^{n}$ with zero mean is determined from (155)

$$
\left\langle\nabla p_{g h}{ }^{n}, \nabla \phi\right\rangle=-\left\langle\frac{\mathbf{n} \cdot\left(\mathbf{g}^{n+1}-\mathbf{g}^{n}\right)}{\Delta t}, \phi\right\rangle_{\Gamma}+\left\langle\frac{h^{n+1}-h^{n}}{\Delta t}, \phi\right\rangle+\nu\left\langle\nabla h^{n+1}, \nabla \phi\right\rangle .
$$

Here

$$
\mathbf{g}^{n}=\frac{1}{\Delta t} \int_{n \Delta t}^{(n+1) \Delta t} \mathbf{g}(t) d t, \quad h^{n}=\frac{1}{\Delta t} \int_{n \Delta t}^{(n+1) \Delta t} h(t) d t
$$

and $\mathbf{f}^{n}$ is determined in the same way from $\mathbf{f}$ as in (73). (This scheme is equivalent to a first-order gauge method-See Appendix C for the proof.)

\subsection{Stability}

We assume the data satisfy (143)-(148) for some given $T>0$. To prove the stability and convergence of the discretiztion scheme, we use $\tilde{\mathbf{u}}$ which satisfies (151) and is bounded in terms of $\left(\mathbf{u}_{\text {in }}, \mathbf{g}\right)$. We define

$$
\tilde{\mathbf{u}}^{n}=\frac{1}{\Delta t} \int_{n \Delta t}^{(n+1) \Delta t} \tilde{\mathbf{u}}(t) d t, \quad \mathbf{v}^{n}=\mathbf{u}^{n}-\tilde{\mathbf{u}}^{n}
$$


and we assume that $\mathbf{u}^{0}=\tilde{\mathbf{u}}^{0}$. Then $\mathbf{v}^{0}=0$ in $\Omega$ and $\mathbf{v}^{n}=0$ on $\Gamma$. We can rewrite (153) as an equation for $\mathbf{v}^{n}$ :

$$
\begin{aligned}
\frac{\mathbf{v}^{n+1}-\mathbf{v}^{n}}{\Delta t}+\mathcal{P}\left(\mathbf{v}^{n} \cdot \nabla \mathbf{v}^{n}-\mathbf{f}^{n}\right) & +\mathcal{P}\left(\mathbf{v}^{n} \cdot \nabla \tilde{\mathbf{u}}^{n}+\tilde{\mathbf{u}}^{n} \cdot \nabla \mathbf{v}^{n}\right) \\
& +\nu(\Delta \mathcal{P}-\mathcal{P} \Delta) \mathbf{v}^{n}=\nu \Delta \mathbf{v}^{n+1}-\tilde{\mathbf{f}}^{n}
\end{aligned}
$$

where

$(158) \quad \tilde{\mathbf{f}}^{n}:=\frac{\tilde{\mathbf{u}}^{n+1}-\tilde{\mathbf{u}}^{n}}{\Delta t}+\nu(\Delta \mathcal{P}-\mathcal{P} \Delta) \tilde{\mathbf{u}}^{n}+\nabla p_{g h}{ }^{n}-\nu \Delta \tilde{\mathbf{u}}^{n+1}+\mathcal{P}\left(\tilde{\mathbf{u}}^{n} \cdot \nabla \tilde{\mathbf{u}}^{n}\right)$.

We claim there exists $C>0$ depending only upon the data such that

$$
\sum_{k=0}^{n-1}\left\|\tilde{\mathbf{f}}^{k}\right\|^{2} \Delta t \leq C
$$

provided $(n+1) \Delta t \leq T$. Because $\tilde{\mathbf{u}} \in V(0, T)^{N} \hookrightarrow C\left([0, T], H^{1}\left(\Omega, \mathbb{R}^{N}\right)\right)$ and

$$
\frac{\tilde{\mathbf{u}}^{n+1}-\tilde{\mathbf{u}}^{n}}{\Delta t}=\int_{t_{n}}^{t_{n+1}} \int_{0}^{\Delta t} \partial_{t} \tilde{\mathbf{u}}(t+s) \frac{d s}{\Delta t} \frac{d t}{\Delta t}=\int_{0}^{2} \partial_{t} \tilde{\mathbf{u}}\left(t_{n}+\tau \Delta t\right) \Lambda(\tau) d \tau
$$

where $\Lambda(\tau)=1-|1-\tau|$, due to the Cauchy-Schwarz inequality we have

$$
\sup _{0 \leq k \leq n}\left\|\nabla \tilde{\mathbf{u}}^{k}\right\|^{2}+\sum_{k=0}^{n}\left\|\tilde{\mathbf{u}}^{k}\right\|_{H^{2}}^{2} \Delta t+\sum_{k=1}^{n-1}\left\|\frac{\tilde{\mathbf{u}}^{n+1}-\tilde{\mathbf{u}}^{n}}{\Delta t}\right\|^{2} \Delta t \leq C .
$$

Using this with (85) bounds the nonlinear term in (158). To bound $\nabla p_{g h}{ }^{n}$, we use Lemma 5 and estimate the time differences in (155) as in (160)-(161) using the Cauchy-Schwarz inequality. Note that the compatibility condition (147) is used to ensure solvability for $p_{g h}{ }^{n}$. Thus we obtain the bound (159).

Following the approach of section 4, we obtain an analog of Theorem 2.

Theorem 5 Let $\Omega$ be a bounded domain in $\mathbb{R}^{N}(N=2$ or 3$)$ with $C^{3}$ boundary, and assume (143)-(148) for some given $T>0$. Consider the time-discrete scheme (153)-(155) with $\mathbf{u}^{0}=\tilde{\mathbf{u}}^{0}$ from (156) and (151). Then there exist positive constants $T_{*}$ and $C_{3}$, such that whenever $n \Delta t \leq T_{*}$, we have

$$
\begin{array}{r}
\sup _{0 \leq k \leq n}\left\|\nabla \mathbf{u}^{k}\right\|^{2}+\sum_{k=0}^{n}\left\|\mathbf{u}^{k}\right\|_{H^{2}}^{2} \Delta t \leq C_{3}, \\
\sum_{k=0}^{n-1}\left(\left\|\frac{\mathbf{u}^{k+1}-\mathbf{u}^{k}}{\Delta t}\right\|^{2}+\left\|\mathbf{u}^{k} \cdot \nabla \mathbf{u}^{k}\right\|^{2}\right) \Delta t \leq C_{3} .
\end{array}
$$

Inequalities (162)-(163) are also true with $\mathbf{u}^{k}$ replaced by $\mathbf{v}^{k}$. 
Proof: We first write (153) as (157). Using (159) and comparing with the proof of Theorem 2, which establishes the stability of the scheme

$\frac{\mathbf{w}^{n+1}-\mathbf{w}^{n}}{\Delta t}+\mathcal{P}\left(\mathbf{w}^{n} \cdot \nabla \mathbf{w}^{n}-\mathbf{f}^{n}\right)+\nu(\Delta \mathcal{P}-\mathcal{P} \Delta) \mathbf{w}^{n}=\nu \Delta \mathbf{w}^{n+1},\left.\quad \mathbf{w}^{n+1}\right|_{\Gamma}=0$,

we see that the only difference is that in (157) we have some extra linear terms of the form

$$
\mathcal{P}(\tilde{\mathbf{u}} \cdot \nabla \mathbf{v}+\mathbf{v} \cdot \nabla \tilde{\mathbf{u}}) .
$$

Similar to (85), we get

$$
\|\mathcal{P}(\tilde{\mathbf{u}} \cdot \nabla \mathbf{v})\|^{2} \leq \varepsilon\|\Delta \mathbf{v}\|^{2}+\frac{C}{\varepsilon}\|\tilde{\mathbf{u}}\|_{H^{1}}^{4}\|\nabla \mathbf{v}\|^{2} .
$$

We estimate the other term in (164) by using Gagliardo-Nirenberg inequalities [Fr, Thm. 10.1] and the Sobolev embeddings of $H^{1}$ into $L^{3}$ and $L^{6}$ :

$$
\|\mathbf{v}\|_{L^{\infty}} \leq \begin{cases}C\|\Delta \mathbf{v}\|_{L^{3 / 2}}^{1 / 2}\|\mathbf{v}\|_{L^{3}}^{1 / 2} \leq C\|\Delta \mathbf{v}\|^{1 / 2}\|\nabla \mathbf{v}\|^{1 / 2} & (N=2), \\ C\|\Delta \mathbf{v}\|^{1 / 2}\|\mathbf{v}\|_{L^{6}}^{1 / 2} \leq C\|\Delta \mathbf{v}\|^{1 / 2}\|\nabla \mathbf{v}\|^{1 / 2} & (N=3) .\end{cases}
$$

Then for $N=2$ and 3 we have

$$
\|\mathcal{P}(\mathbf{v} \cdot \nabla \tilde{\mathbf{u}})\|^{2} \leq\|\mathbf{v}\|_{L^{\infty}}^{2}\|\nabla \tilde{\mathbf{u}}\|^{2} \leq \varepsilon\|\Delta \mathbf{v}\|^{2}+\frac{C}{\varepsilon}\|\tilde{\mathbf{u}}\|_{H^{1}}^{4}\|\nabla \mathbf{v}\|^{2} .
$$

With these estimates, the rest of the proof of the stability of $\mathbf{v}^{n}$ is essentially the same as that of Theorem 2 and therefore we omit the details. The stability of $\mathbf{v}^{n}$ leads to that of $\mathbf{u}^{n}$, using (161), (165) and (167).

\subsection{Convergence}

First, we define $\mathbf{u}_{\Delta t}$ and $\mathbf{U}_{\Delta t}$ exactly as before by (99) and (100), and define

$$
p_{g h \Delta t}(t)=p_{g h}{ }^{n} \quad \text { for } t \in\left[t_{n}, t_{n+1}\right), n=0,1, \ldots
$$

Then, (153) can be written as

$$
\begin{array}{r}
\partial_{t} \mathbf{u}_{\Delta t}+\mathcal{P}\left(\mathbf{U}_{\Delta t} \cdot \nabla \mathbf{U}_{\Delta t}-\mathbf{f}_{\Delta t}-\nu \Delta \mathbf{U}_{\Delta t}\right)+\nabla p_{g h} \Delta t \\
=\nu \Delta\left(\mathbf{U}_{\Delta t}(\cdot+\Delta t)-\mathbf{U}_{\Delta t}\right)+\nu \nabla \nabla \cdot \mathbf{U}_{\Delta t},
\end{array}
$$

Comparing with (101), we see that exactly as before in the proof of Theorem 3 , we are justified to pass to the limit along some subsequence $\Delta t_{j} \rightarrow 0$, in all the linear terms except $\nabla p_{g h}{ }_{\Delta t}$.

In fact we claim that with $Q=\Omega \times(0, T)$,

$$
\left\|\nabla p_{g h \Delta t}-\nabla p_{g h}\right\|_{L^{2}(Q)} \rightarrow 0 \quad \text { as } \Delta t \rightarrow 0 .
$$


The proof is straightforward using Lemma 5. First, let $h_{\Delta t}(t)=h^{n+1}$ for $t \in\left[t_{n}, t_{n+1}\right), n=0,1, \ldots$. As in (104) we have $\nabla h_{\Delta t} \rightarrow \nabla h$ strongly in $L^{2}(Q)$.

Next, suppose generally that $X$ is a Banach space and $\psi \in L^{2}(0, T ; X)$. Let

$$
\psi_{\Delta t}(t)=\int_{0}^{2} \psi\left(t_{n}+\tau \Delta t\right) \Lambda(\tau) d \tau, \quad t \in\left[t_{n}, t_{n+1}\right), n=0,1, \ldots,
$$

as in (160). (Extend $\psi$ by zero for $t>T$.) The map $\psi \rightarrow \psi_{\Delta t}$ is bounded on $L^{2}(0, T ; X)$ by the Cauchy-Schwarz inequality, and $\psi_{\Delta t} \rightarrow \psi$ strongly as $\Delta t \rightarrow 0$, since this is true for $\psi$ in $C([0, T], X)$, a dense set.

Applying this result with $\psi=\partial_{t} h, X=\left(H^{1}\right)^{\prime}$, and with $\psi=\partial_{t}(\mathbf{n} \cdot \mathbf{g})$, $X=H^{-1 / 2}(\Gamma)$, comparing with (160) and using Lemma 5, we get the strong convergence asserted in (170).

As before in the proof of Theorem 3, we can now pass to the limit in all terms of (169) and find that

$$
\mathbf{u}_{\Delta t} \rightarrow \mathbf{u} \quad \text { weakly in } V\left(0, T_{*}\right)^{N},
$$

where $\mathbf{u}$ satisfies (132), which is equivalent to (136) of our unconstrained formulation. It remains to verify the boundary and initial conditions, and study the regularity of $\nabla \cdot \mathbf{u}$.

Recall $\mathbf{u}_{\Delta t}=\mathbf{v}_{\Delta t}+\tilde{\mathbf{u}}_{\Delta t}$ with $\mathbf{v}_{\Delta t}$ and $\tilde{\mathbf{u}}_{\Delta t}$ defined as in (99) by linear interpolation. From Theorem 5 , we know $\mathbf{v}_{\Delta t}$ is uniformly bounded in

$$
V_{\mathrm{b}}:=V\left(0, T_{*}\right)^{N} \cap\left\{\mathbf{w}|\mathbf{w}(t=0)=0, \mathbf{w}|_{\Gamma}=0\right\},
$$

which is a Hilbert space carrying zero boundary and initial conditions. Consequently $\mathbf{v}_{\Delta t_{j}} \rightarrow \mathbf{v}$ weakly in $V_{\mathrm{b}}$, and $\mathbf{v}=0$ at $t=0$ and on $\Gamma$. Next we note that

$$
\left\|\tilde{\mathbf{u}}_{\Delta t}-\tilde{\mathbf{u}}\right\|_{L^{2}(Q)} \rightarrow 0 \quad \text { as } \Delta t \rightarrow 0
$$

This follows because $\tilde{\mathbf{u}}_{\Delta t}$ is bounded in $V(0, T)^{N} \hookrightarrow H^{1}(Q)^{N}$ by $(161)$, and the embedding $H^{1}(Q) \hookrightarrow L^{2}(Q)$ is compact. We already know $\tilde{\mathbf{u}} \in V(0, T)^{N}$ with $\left.\tilde{\mathbf{u}}\right|_{\Gamma}=\mathbf{g}$ and $\tilde{\mathbf{u}}(t=0)=\mathbf{u}_{\text {in }}$. Hence we have $\mathbf{u}=\mathbf{v}+\tilde{\mathbf{u}}$ and it satisfies the correct boundary and initial conditions. By Lemma 6 we have $\mathbf{u} \in W_{\operatorname{div}}\left(0, T_{*}\right)$.

As a by-product of this convergence analysis for the scheme (153)-(154), we get the following existence and uniqueness theorem. Convergence of $\mathbf{u}_{\Delta t}$ to $\mathbf{u}$ along any subsequence follows from the uniqueness.

Theorem 6 Let $\Omega$ be a bounded, connected domain in $\mathbb{R}^{N}(N=2$ or 3$)$ and assume (143)-(148). Then there exists $T_{*}>0$ so that a unique strong solution of (135)-(136) and (130)-(131) exists on $\left[0, T_{*}\right]$, with $\mathbf{u} \in W_{\mathrm{div}}\left(0, T_{*}\right)$. Moreover, $\nabla \cdot \mathbf{u}-h \in W(0, T)$ is a smooth solution of the heat equation for $t>0$ with no-flux boundary conditions. The map $t \mapsto\|\nabla \cdot \mathbf{u}-h\|^{2}$ is smooth for $t>0$ and we have the dissipation identity

$$
\frac{d}{d t} \frac{1}{2}\|\nabla \cdot \mathbf{u}-h\|^{2}+\nu\|\nabla(\nabla \cdot \mathbf{u}-h)\|^{2}=0 .
$$


If we further assume $h \in V(0, T)$ and $\nabla \cdot \mathbf{u}_{\text {in }} \in H^{1}(\Omega)$, then $\mathbf{u} \in V_{\text {div }}\left(0, T_{*}\right)$.

The approximations $\mathbf{u}_{\Delta t}$ constructed above converge to $\mathbf{u}$ weakly in $V\left(0, T_{*}\right)^{N}$ and strongly in $L^{2}\left(\left[0, T_{*}\right] \times \Omega, \mathbb{R}^{N}\right)$.

Proof: The existence of $\mathbf{u} \in W_{\mathrm{div}}\left(0, T_{*}\right)$ has been proved, and we can prove the uniqueness by the same method as in Theorem 3. We study $w=\nabla \cdot \mathbf{u}-h$ in a manner similar to the proof of Theorem 3. Combining (136) with (135), we get (134) for any $\phi \in H^{1}(\Omega)$. With $w=\nabla \cdot \mathbf{u}-h$, taking $\phi \in D(A)$ as in (108) we have

$$
\langle w, \phi\rangle=\langle\mathbf{n} \cdot \mathbf{g}, \phi\rangle_{\Gamma}-\langle\mathbf{u}, \nabla \phi\rangle-\langle h, \phi\rangle .
$$

Therefore $t \mapsto\langle w, \phi\rangle$ is absolutely continuous, and (134) yields $(d / d t)\langle w, \phi\rangle=$ $\langle w, A \phi\rangle$ for a.e. $t$. This means $w$ is a weak solution in the sense of Ball [Ba], and the rest of the proof that $w$ is a smooth solution of the heat equation satisfying (174) goes as before, using (147) to get $\int_{\Omega} w=0$.

If we further assume $h \in V(0, T)$ and $\nabla \cdot \mathbf{u}_{\text {in }} \in H^{1}(\Omega)$, then $w(0) \in H^{1}(\Omega)$. We claim

$$
H^{1}(\Omega)=D\left((-A)^{1 / 2}\right) .
$$

Then semigroup theory yields $w \in C\left(\left[0, T_{*}\right], D\left((-A)^{1 / 2}\right)\right)$, so since

$$
0=\left\langle-\Delta w, \partial_{t} w-\nu \Delta w\right\rangle=\frac{d}{d t} \frac{1}{2}\|\nabla w\|^{2}+\nu\|\Delta w\|^{2}
$$

for $t>0$, we deduce $w \in V\left(0, T_{*}\right)$, and $\nabla \cdot \mathbf{u}$ is in the same space.

To prove (176), note $X:=D\left((-A)^{1 / 2}\right)$ is the closure of $D(A)$ from (108) in the norm given by

$$
\|w\|_{X}^{2}=\|w\|^{2}+\left\|(-A)^{1 / 2} w\right\|^{2}=\langle(I-\nu \Delta) w, w\rangle=\int_{\Omega}|w|^{2}+\nu|\nabla w|^{2} .
$$

Clearly $X \subset H^{1}(\Omega)$. For the other direction, let $w \in H^{1}(\Omega)$ be arbitrary. We may suppose $w \in C^{\infty}(\bar{\Omega})$ since this space is dense in $H^{1}(\Omega)$. Now we only need to construct a sequence of $C^{2}$ functions $w_{n} \rightarrow 0$ in $H^{1}$ norm with $\mathbf{n} \cdot \nabla w_{n}=\mathbf{n} \cdot \nabla w$ on $\Gamma$. This is easily accomplished using functions of the form $w_{n}(x)=\xi_{n}(\operatorname{dist}(x, \Gamma)) \mathbf{n} \cdot \nabla w(x)$, where $\xi_{n}(s)=\xi(n s) / n$ with $\xi$ smooth and satisfying $\xi(0)=0, \xi^{\prime}(0)=1$ and $\xi(s)=0$ for $s>1$. This proves (176). 


\section{Estimate on the divergence}

We can provide an estimate on $\nabla \cdot \mathbf{u}^{n}-h^{n}$ for the time-discrete scheme in section 7. For this we prove another estimate on Stokes pressure, showing that its $L^{2}$ norm is controlled by the tangential components of vorticity on the boundary.

Lemma 7 ( $L^{2}$ estimate for Stokes pressure) Let $\Omega \subset \mathbb{R}^{N}(N \geq 2)$ be a bounded connected domain with $C^{2}$ boundary. Then there is a constant $C>0$ so that for any $\mathbf{u} \in H^{2}\left(\Omega, \mathbb{R}^{N}\right)$, the associated Stokes pressure $p_{S} \in H^{1}(\Omega)$ defined by $\nabla p_{S}=(\Delta \mathcal{P}-\mathcal{P} \Delta) \mathbf{u}$ with zero mean satisfies

$$
\left\|p_{S}\right\| \leq C\left\|\mathbf{n} \cdot\left(\nabla \mathbf{u}-\nabla \mathbf{u}^{T}\right)\right\|_{L^{2}(\Gamma)} \leq C^{2}\|\mathbf{u}\|^{1 / 4}\|\mathbf{u}\|_{H^{2}(\Omega)}^{3 / 4} .
$$

Proof: For any $\phi \in L^{2}(\Omega)$, define $\psi$ with mean zero by

$$
\Delta \psi=\phi-\bar{\phi},\left.\quad \mathbf{n} \cdot \nabla \psi\right|_{\Gamma}=0,
$$

where $\bar{\phi}$ is the mean value of $\phi$ over $\Omega$. Then, since $p_{\mathrm{S}}$ has mean zero, we have

$$
\left\langle p_{\mathrm{S}}, \phi\right\rangle=\left\langle p_{\mathrm{S}}, \phi-\bar{\phi}\right\rangle=\left\langle p_{\mathrm{S}}, \Delta \psi\right\rangle=-\left\langle\nabla p_{\mathrm{S}}, \nabla \psi\right\rangle \text {. }
$$

From (50), we get

$$
\left|\left\langle p_{\mathrm{S}}, \phi\right\rangle\right| \leq c_{0}\left\|\mathbf{n} \cdot\left(\nabla \mathbf{u}-\nabla \mathbf{u}^{T}\right)\right\|_{L^{2}(\Gamma)}\|\nabla \psi\|_{L^{2}(\Gamma)}
$$

By elliptic regularity theory for (179),

$$
\|\nabla \psi\|_{L^{2}(\Gamma)} \leq c_{1}\|\psi\|_{H^{3 / 2}(\Omega)} \leq c_{2}\|\phi-\bar{\phi}\| \leq c_{2}\|\phi\| .
$$

Taking $\phi=p_{\mathrm{S}}$ in (181) gives the first inequality in the Lemma, and the second follows by standard trace and interpolation theorems.

Proposition 3 Make the same assumptions as in Theorem 5, and let

$$
w^{n}=\nabla \cdot \mathbf{u}^{n}-h^{n}, \quad n=0,1, \ldots
$$

Then there exists $C>0$ such that as long as $n \Delta t \leq T_{*}$ we have

$$
\sup _{0 \leq k \leq n}\left\|w^{k}\right\|_{H^{1}(\Omega)^{\prime}}^{2}+\sum_{k=1}^{n}\left\|w^{k}\right\|^{2} \Delta t \leq C\left(\left\|w^{0}\right\|_{H^{1}(\Omega)^{\prime}}^{2}+\Delta t^{1 / 2}\right) .
$$

Proof: For $\phi \in H^{1}(\Omega)$ with mean zero, we compute from (153)-(155) that

$$
\left\langle\frac{w^{n+1}-w^{n}}{\Delta t}, \phi\right\rangle+\nu\left\langle\nabla\left(w^{n+1}+p_{\mathrm{S}}^{n+1}-p_{\mathrm{S}}^{n}\right), \nabla \phi\right\rangle=0 .
$$

Here we used Lemma 1 to say $(I-\mathcal{P}) \Delta \mathbf{u}^{n+1}=\nabla \nabla \cdot \mathbf{u}^{n+1}+(\Delta \mathcal{P}-\mathcal{P} \Delta) \mathbf{u}^{n+1}$. Next, note that $\int_{\Omega} w^{n}=0$ by the compatibility condition (147). We let $q^{n}$ be the mean-zero solution of

$$
-\Delta q^{n}=w^{n}, \quad \mathbf{n} \cdot \nabla q^{n}=0 \text { on } \Gamma .
$$


Note that $\left\|\nabla q^{n}\right\|$ is equivalent to $\left\|w^{n}\right\|_{H^{1}(\Omega)^{\prime}}$. Taking $\phi=q^{n+1}$ in (183), we find

$$
\left\langle\frac{\nabla q^{n+1}-\nabla q^{n}}{\Delta t}, \nabla q^{n+1}\right\rangle+\nu\left\langle w^{n+1}+p_{\mathrm{S}}^{n+1}-p_{\mathrm{S}}^{n}, w^{n+1}\right\rangle=0,
$$

whence

$$
\frac{\left\|\nabla q^{n+1}\right\|^{2}-\left\|\nabla q^{n}\right\|^{2}}{\Delta t}+2 \nu\left\|w^{n+1}\right\|^{2} \leq \nu\left(\left\|w^{n+1}\right\|^{2}+\left\|p_{\mathrm{S}}^{n+1}-p_{\mathrm{S}}^{n}\right\|^{2}\right),
$$

and

$$
\left\|\nabla q^{n}\right\|^{2}+\nu \sum_{k=0}^{n-1}\left\|w^{k+1}\right\|^{2} \Delta t \leq\left\|\nabla q^{0}\right\|^{2}+\nu \sum_{k=0}^{n-1}\left\|p_{\mathrm{S}}^{k+1}-p_{\mathrm{S}}^{k}\right\|^{2} \Delta t .
$$

Since $\nabla\left(p_{\mathrm{S}}^{n+1}-p_{\mathrm{S}}^{n}\right)=(\Delta \mathcal{P}-\mathcal{P} \Delta)\left(\mathbf{u}^{n+1}-\mathbf{u}^{n}\right)$, we use Lemma 7 to infer

$$
\left\|p_{\mathrm{S}}^{n+1}-p_{\mathrm{S}}^{n}\right\|^{2} \leq C\left\|\mathbf{u}^{n+1}-\mathbf{u}^{n}\right\|^{1 / 2}\left\|\mathbf{u}^{n+1}-\mathbf{u}^{n}\right\|_{H^{2}}^{3 / 2} .
$$

Then by Hölder's inequality and Theorem 5,

$$
\begin{aligned}
\sum_{k=0}^{n-1}\left\|p_{\mathrm{S}}^{k+1}-p_{\mathrm{S}}^{k}\right\|^{2} \Delta t & \leq C\left[\sum_{k=0}^{n-1}\left\|\mathbf{u}^{k+1}-\mathbf{u}^{k}\right\|^{2} \Delta t\right]^{\frac{1}{4}}\left[\sum_{k=0}^{n-1}\left\|\mathbf{u}^{k+1}-\mathbf{u}^{k}\right\|_{H^{2}}^{2} \Delta t\right]^{\frac{3}{4}} \\
& \leq C \sqrt{\Delta t} .
\end{aligned}
$$

\section{Acknowledgments}

The fact (related to Lemma 2) that $\mathbf{n} \cdot \nabla p \in L^{2}\left(\Omega_{s}\right)$ implies $\nabla p \in L^{2}(\Omega)$ for harmonic $p$ was proved several years ago by Oscar Gonzalez and RLP (unpublished) through a partitioning and flattening argument. RLP is grateful for this collaboration. The authors thank Stefan Müller for discussions leading to (69), which improves the key constant in Theorem 1 from $\frac{2}{3}+\varepsilon$ to $\frac{1}{2}+\varepsilon$. We also thank Stefan Luckhaus for pointing out an argument of Sanni [Sa] which substantially simplified the proof of the integrated Neumann-to-Dirichlet estimate that we use from Lemma 2.

This material is based upon work supported by the National Science Foundation under grant nos. DMS 03-05985 and 04-05343 (RLP) and DMS 01-07218 and 05-12176 (JGL). JGL and RLP are thankful for the support of the Institute for Mathematical Sciences at the National University of Singapore. RLP acknowledges support by the Distinguished Ordway Visitors Program of the School of Mathematics and the Institute for Mathematics and its Applications at the University of Minnesota, and through a Mercator Professorship awarded by the Deutsche Forschungsgemeinschaft. 


\section{A Optimal commutator estimate in a half space}

Consider $\Omega$ to be the half space $\mathbb{R}_{+}^{N}$ where $x_{N}>0$. In this case, one must take care to define the Helmholtz projection, since $\nabla H^{1}(\Omega)$ is now not closed in $L^{2}\left(\Omega, \mathbb{R}^{N}\right)$ (cf. [Soh] $)$. Let $B \subset \Omega$ be a fixed bounded domain and let $Y$ be the space of functions $q \in L_{\text {loc }}^{2}(\Omega)$ such that $\int_{B} q=0$ and $\nabla q \in L^{2}\left(\Omega, \mathbb{R}^{N}\right)$. Then $Y$ is a Hilbert space with norm $\|q\|_{Y}^{2}=\int_{\Omega}^{B}|\nabla q|^{2}$ and $\nabla Y$ is a closed subspace of $L^{2}\left(\Omega, \mathbb{R}^{N}\right)$. The Helmholtz projection $\mathcal{P}$ may be defined as in (7) with $q \in Y$ instead of $H^{1}(\Omega)$.

We note that smooth $q \in C^{\infty}(\bar{\Omega})$ with bounded support are dense in $Y$. The proof is similar to that in the case $\Omega=\mathbb{R}^{N}$ as given in [Soh, Lemma 2.5.4] approximate by functions with bounded support via cutoff, then mollify. Here one should shift slightly before mollifying, replacing $q(x)$ by $q\left(x-\varepsilon \mathbf{e}_{N}\right)$, as in standard proofs of the density of smooth functions in Sobolev spaces (cf. [Ev]).

If the estimate (15) holds for some $\varepsilon>0, C \geq 0$ independent of $\mathbf{u}$, then a simple scaling argument shows that it holds with $C=0$. We claim that in fact estimate (15) holds with $\varepsilon=0$ and $C=0$, and is sharp in general.

Theorem 7 Let $\Omega=\mathbb{R}_{+}^{N}$ with $N \geq 2$. Then

$$
\int_{\Omega}|(\Delta \mathcal{P}-\mathcal{P} \Delta) \mathbf{u}|^{2} \leq \frac{1}{2} \int_{\Omega}|\Delta \mathbf{u}|^{2}
$$

for all $\mathbf{u} \in H^{2} \cap H_{0}^{1}\left(\Omega, \mathbb{R}^{N}\right)$, and equality holds for some $\mathbf{u}$.

Proof: It suffices to study $\mathbf{u}$ in a dense subset of $H^{2} \cap H_{0}^{1}\left(\Omega, \mathbb{R}^{N}\right)$, so we take $\mathbf{u}$ smooth in $\overline{\mathbb{R}_{+}^{N}}$ with bounded support. First we obtain an explicit expression for the Fourier transform of $\nabla p_{\mathrm{S}}=(\Delta \mathcal{P}-\mathcal{P} \Delta) \mathbf{u}$ using the boundary value problem (21) for $p=p_{\mathrm{S}}$. Since $\mathbf{u}=0$ for $x_{N}=0$ and $\mathbf{n}=\mathbf{e}_{N}=(0, \ldots, 0,-1)$, we have $\mathbf{n} \cdot(\Delta \mathbf{u}-\nabla \nabla \cdot \mathbf{u})=\partial_{N} \nabla \cdot u_{\|}$on $\Gamma$, where $u_{\|}=\left(u_{1}, \ldots, u_{N-1}\right)$. Formally taking the Fourier transform in tangential variables, for $k \in \mathbb{R}^{N-1}$ and $s=x_{N}$ we need

$$
\left(\partial_{s}^{2}-|k|^{2}\right) \hat{p}(k, s)=0, \quad \partial_{s} \hat{p}(k, 0)=-i k \cdot \partial_{s} \hat{u}_{\|}(k, 0) .
$$

Since $\hat{p}$ should not grow exponentially in $s>0$, for $k \neq 0$ we get the formula

$$
\hat{p}(k, s)=a(k) e^{-|k| s} \quad \text { where } a(k)=i \hat{k} \cdot \partial_{s} \hat{u}_{\|}(k, 0), \quad \hat{k}=k /|k| .
$$

Since $a(k)$ is rapidly decreasing, inversion yields a smooth $p$ on $\overline{\mathbb{R}_{+}^{N}}$ with

$$
\widehat{\nabla p}(k, s)=(i k,-|k|) a(k) e^{-|k| s},
$$

$$
\int_{\Omega}|\nabla p|^{2}=\int_{\mathbb{R}^{N-1}} \int_{0}^{\infty}\left(|k|^{2}|\hat{p}|^{2}+\left|\partial_{s} \hat{p}\right|^{2}\right) d s d k=\int_{\mathbb{R}^{N-1}}|k||a(k)|^{2} d k<\infty
$$

Hence (188) determines a harmonic function $p \in Y$ (adjusting by a constant if necessary). Fourier inversion of the last component of (189) shows that the 
boundary condition in (21) holds, whence (20) holds for all smooth $\phi \in Y$ with bounded support. Hence (188) indeed determines the Stokes pressure.

Now,

$$
\int_{\Omega}|\Delta \mathbf{u}|^{2}=\int_{\Omega}\left|\Delta u_{N}\right|^{2}+\int_{\mathbb{R}^{N-1}} \int_{0}^{\infty}\left|\left(\partial_{s}^{2}-|k|^{2}\right) \hat{u}_{\|}\right|^{2} d s d k .
$$

Let $v(s)=\hat{k} \cdot \hat{u}_{\|}(k, s), w(s)=e^{-|k| s}$. Then since $v(0)=0, w(0)=1$ and $w^{\prime \prime}=|k|^{2} w$, by Green's identity and Cauchy-Schwarz we find

$$
\begin{aligned}
& |a(k)|^{2}=\left|v^{\prime}(0)\right|^{2}=\left|\int_{0}^{\infty}\left(w v^{\prime \prime}-w^{\prime \prime} v\right) d s\right|^{2} \\
& \quad \leq \int_{0}^{\infty} w(s)^{2} d s \int_{0}^{\infty}\left|\left(\partial_{s}^{2}-|k|^{2}\right) v\right|^{2} d s=\frac{1}{2|k|} \int_{0}^{\infty}\left|\left(\partial_{s}^{2}-|k|^{2}\right) v\right|^{2} d s .
\end{aligned}
$$

Together with (191) and (190) this implies

$$
\int_{\Omega}|\nabla p|^{2} \leq \frac{1}{2} \int_{\Omega}|\Delta \mathbf{u}|^{2}
$$

To see that equality is possible, choose $a(k)$ smooth with compact support and zero near $k=0$, and determine $\mathbf{u} \in H^{2} \cap H_{0}^{1}\left(\Omega, \mathbb{R}^{N}\right)$ so $u_{N}=0$ and

$$
\hat{u}_{\|}(k, s)=s e^{-|k| s}(-i \hat{k}) a(k) \text {. }
$$

One computes then that $\left|\left(\partial_{s}^{2}-k^{2}\right) \hat{u}_{\|}\right|=e^{-|k| s}$, and equality holds in (192), while (187) and (188) hold.

\section{B Range of the commutator $\Delta \mathcal{P}-\mathcal{P} \Delta$}

According to (19) the range of the commutator $[\Delta, \mathcal{P}]$ acting on $H^{2}\left(\Omega, \mathbb{R}^{N}\right)$ can be characterized as the space of gradients of Stokes pressures. By (20), the space of Stokes pressures is given by

$$
\mathcal{S}_{p}:=\left\{p \in H^{1}(\Omega) / \mathbb{R} \mid \Delta p=0 \text { in } \Omega \text { and }\left.\mathbf{n} \cdot \nabla p\right|_{\Gamma} \in \mathcal{S}_{\Gamma}\right\},
$$

where $\mathcal{S}_{\Gamma}$ is the subspace of $H^{-1 / 2}(\Gamma)$ given by

$$
\mathcal{S}_{\Gamma}:=\left\{f=\left.\mathbf{n} \cdot(\Delta-\nabla \nabla \cdot) \mathbf{u}\right|_{\Gamma} \mid \mathbf{u} \in H^{2}\left(\Omega, \mathbb{R}^{N}\right)\right\} .
$$

The Stokes pressure $p$ with zero average is determined uniquely by $f=\left.\mathbf{n} \cdot \nabla p\right|_{\Gamma} \in$ $\mathcal{S}_{\Gamma}$, with $\|p\|_{H^{1}(\Omega)} \leq C\|f\|_{H^{-1 / 2}(\Gamma)}$ by the Lax-Milgram lemma.

The space $\mathcal{S}_{\Gamma}$ may be characterized as follows.

Theorem 8 Assume $\Omega \subset \mathbb{R}^{N}$ is a bounded, connected domain and its boundary $\Gamma$ is of class $C^{3}$. Denote the connected components of $\Gamma$ by $\Gamma_{i}, i=1, \ldots, m$. Then

$$
\begin{aligned}
\mathcal{S}_{\Gamma} & =\left\{f \in H^{-1 / 2}(\Gamma) \mid \int_{\Gamma_{i}} f=0 \text { for } i=1, \ldots, m\right\} \\
& =\left\{f=\left.\mathbf{n} \cdot(\Delta-\nabla \nabla \cdot) \mathbf{u}\right|_{\Gamma} \mid \mathbf{u} \in H^{2} \cap H_{0}^{1}\left(\Omega, \mathbb{R}^{N}\right)\right\},
\end{aligned}
$$


and moreover, the map $\left.\mathbf{u} \mapsto \mathbf{n} \cdot(\Delta-\nabla \nabla \cdot) \mathbf{u}\right|_{\Gamma}$ from $H^{2} \cap H_{0}^{1}\left(\Omega, \mathbb{R}^{N}\right)$ to $\mathcal{S}_{\Gamma}$ admits a bounded right inverse.

Proof: First we check that if $f=\left.\mathbf{n} \cdot(\Delta-\nabla \nabla \cdot) \mathbf{u}\right|_{\Gamma}$ with $\mathbf{u} \in H^{2}\left(\Omega, \mathbb{R}^{N}\right)$, then $\int_{\Gamma_{i}} f=0$ for $i=1, \ldots, m$. For each $\Gamma_{i}$, there is an $s_{i}>0$ small enough and a smooth cut-off function $\rho_{i}$ defined in $\Omega$ which satisfies $\rho_{i}(x)=1$ when $\operatorname{dist}\left(x, \Gamma_{i}\right)<s_{i}$ and $\rho_{i}(x)=0$ when $\operatorname{dist}\left(x, \Gamma_{j}\right)<s_{i}$ for all $j \neq i$. Let $\mathbf{a}=$ $(\Delta-\nabla \nabla \cdot)\left(\rho_{i} \mathbf{u}\right)$. Then $\mathbf{a} \in L^{2}\left(\Omega, \mathbb{R}^{N}\right)$ and $\nabla \cdot \mathbf{a}=0$, so

$$
\int_{\Gamma_{i}} f=\int_{\Gamma} \mathbf{n} \cdot \mathbf{a}=\int_{\Omega} \nabla \cdot \mathbf{a}=0 .
$$

Next, let $f \in H^{-1 / 2}(\Gamma)$ with $\int_{\Gamma_{i}} f=0$ for all $i$. We will show that we can find some $\mathbf{u} \in H^{2} \cap H_{0}^{1}\left(\Omega, \mathbb{R}^{N}\right)$ so that $f=\left.\mathbf{n} \cdot(\Delta-\nabla \nabla \cdot) \mathbf{u}\right|_{\Gamma}$. First, treating each boundary component separately, we can solve the problem

$$
\Delta_{\Gamma} \psi=-f \quad \text { on } \Gamma, \quad \int_{\Gamma_{i}} \psi=0 \text { for } i=1, \ldots, m,
$$

where $\Delta_{\Gamma}$ is the (positive) Laplace-Beltrami operator on $\Gamma$. Denote the mapping $f \mapsto \psi$ by $T$. Then $T: H^{-1}(\Gamma) \rightarrow H^{1}(\Gamma)$ is bounded ([Au, theorem 1.71, theorem 4.7], [Tay, p. 306, Proposition 1.6]). Also $T: L^{2}(\Gamma) \rightarrow H^{2}(\Gamma)$ is bounded, by elliptic regularity theory [Tay, p. 306, Proposition 1.6]. So, interpolation implies (see [LM, vol I, p. 37, Remark 7.6])

$$
\|\psi\|_{H^{3 / 2}(\Gamma)} \leq C\|f\|_{H^{-1 / 2}(\Gamma)} .
$$

Now by an inverse trace theorem [RR, Theorem 6.109], there exists a map $\psi \mapsto q \in H^{3}(\Omega)$ with

$$
q=0 \text { and } \mathbf{n} \cdot \nabla q=\psi \quad \text { on } \Gamma, \quad\|q\|_{H^{3}(\Omega)} \leq C\|\psi\|_{H^{3 / 2}(\Gamma)} .
$$

We may assume $q$ is supported in a small neighborhood of $\Gamma$. Define

$$
\mathbf{u}=\left(I-\mathbf{n n}^{T}\right) \nabla q .
$$

Then $f \mapsto \mathbf{u}$ is bounded from $\mathcal{S}_{\Gamma}$ to $H^{2} \cap H_{0}^{1}\left(\Omega, \mathbb{R}^{N}\right)$. We claim

$$
\mathbf{n} \cdot(\Delta-\nabla \nabla \cdot) \mathbf{u}=f \quad \text { on } \Gamma .
$$

The proof of this claim amounts to showing that the derivative along the normal $\mathbf{n} \cdot \nabla$ and normal projection $\mathbf{n n}^{T}$ commute on the boundary with the tangential gradient and divergence operators $\left(I-\mathbf{n n}^{T}\right) \nabla$ and $\nabla \cdot\left(I-\mathbf{n n}^{T}\right)$ for the functions involved.

First, since $\mathbf{n} \cdot \mathbf{u}=0$, by expanding $\Delta(\mathbf{n} \cdot \mathbf{u})$ we get

$$
\mathbf{n} \cdot \Delta \mathbf{u}=-(\Delta \mathbf{n}) \cdot \mathbf{u}-2 \nabla \mathbf{n}: \nabla \mathbf{u}=0 \quad \text { on } \Gamma,
$$


since for each $i, \nabla n_{i}$ is tangential and $\nabla u_{i}$ is normal to $\Gamma$ - indeed, using $\partial_{j} n_{i}=\partial_{i} n_{j}$ and (48) and (49), we have that

$$
\nabla \mathbf{n}: \nabla \mathbf{u}=\left(\partial_{j} n_{i}\right)\left(\partial_{j} u_{i}\right)=\left(\partial_{i} n_{j}\right)\left(n_{j} n_{k} \partial_{k} u_{i}\right)=0 \quad \text { on } \Gamma .
$$

Next we calculate in $\Omega$ that

$$
\mathbf{n} \cdot \nabla \nabla \cdot \mathbf{u}=\nabla \cdot(\mathbf{n} \cdot \nabla \mathbf{u})-\nabla \mathbf{n}: \nabla \mathbf{u}
$$

Note that $\mathbf{n} \cdot \nabla\left(\mathbf{n n}^{T}\right)=0$ by (48), so $\mathbf{n} \cdot \nabla$ commutes with $I-\mathbf{n n}^{T}$ in $\Omega$. Then since $\mathbf{u}=\left(I-\mathbf{n n}^{T}\right) \mathbf{u}$ from (199) we get

$$
\mathbf{n} \cdot \nabla \mathbf{u}=\left(I-\mathbf{n n}^{T}\right)(\mathbf{n} \cdot \nabla) \mathbf{u}=\left(I-\mathbf{n n}^{T}\right)(\mathbf{n} \cdot \nabla) \nabla q .
$$

Now

$$
(\mathbf{n} \cdot \nabla) \nabla q=\nabla(\mathbf{n} \cdot \nabla q)-\mathbf{a}
$$

where

$$
a_{i}=\left(\partial_{i} n_{j}\right)\left(\partial_{j} q\right)=\left(\partial_{j} n_{i}\right)\left(\partial_{j} q\right)
$$

This quantity lies in $H^{2}(\Omega)$ and vanishes on $\Gamma$ since $\nabla q=\left(\mathbf{n n}^{T}\right) \nabla q$ on $\Gamma$. (This can be proved by approximation.) Using part (i) of Lemma 3, we have that $\nabla \cdot\left(I-\mathbf{n n}^{T}\right) \mathbf{a}=0$ on $\Gamma$. Combining (201)-(205) we conclude that

$$
\mathbf{n} \cdot(\Delta-\nabla \nabla \cdot) \mathbf{u}=-\nabla \cdot\left(I-\mathbf{n n}^{T}\right) \nabla(\mathbf{n} \cdot \nabla q) \quad \text { on } \Gamma .
$$

But it is well known that at any point $x$ where $\Phi(x)=r \in(0, s)$, for any smooth function $\phi$ on $\Omega_{s}$,

$$
\nabla \cdot\left(I-\mathbf{n n}^{T}\right) \nabla \phi=\Delta \phi-(\nabla \cdot \mathbf{n})(\mathbf{n} \cdot \nabla \phi)-(\mathbf{n} \cdot \nabla)^{2} \phi=-\Delta_{\Gamma_{r}}\left(\left.\phi\right|_{\Gamma_{r}}\right) .
$$

where $\Delta_{\Gamma_{r}}$ is the Laplace-Beltrami operator on $\Gamma_{r}$. So taking $r \rightarrow 0$ we see that the right hand side of (207) is exactly $-\Delta_{\Gamma}\left(\left.\mathbf{n} \cdot \nabla q\right|_{\Gamma}\right)$. So by (196) and (198) we have established the claim in (200). This finishes the proof.

Remark 3. Given a velocity field $\mathbf{u} \in H^{2} \cap H_{0}^{1}\left(\Omega, \mathbb{R}^{3}\right)$, the associated Stokes pressure is determined by the normal component at the boundary of the curl of the vorticity $\omega=\nabla \times \mathbf{u}$, which is a vector field in $H^{1}\left(\Omega, \mathbb{R}^{3}\right)$. A question related to Theorem 8 is whether the space $\mathcal{S}_{\Gamma}$ of such boundary values $\mathbf{n} \cdot \nabla \times \omega$ is constrained in any way, as compared to the space of boundary values $\mathbf{n} \cdot \nabla \times \mathbf{v}$ where $\mathbf{v} \in H^{1}\left(\Omega, \mathbb{R}^{3}\right)$ is arbitrary.

The answer is no. In [Te1, Appendix I, Proposition 1.3], Temam proves

$$
\nabla \times H^{1}\left(\Omega, \mathbb{R}^{3}\right)=\left\{\mathbf{g} \in L^{2}\left(\Omega, \mathbb{R}^{3}\right) \mid \nabla \cdot \mathbf{g}=0, \int_{\Gamma_{i}} \mathbf{n} \cdot \mathbf{g}=0 \forall i\right\} .
$$

Clearly $\mathcal{S}_{\Gamma} \subset \mathbf{n} \cdot \nabla \times H^{1}\left(\Omega, \mathbb{R}^{3}\right)$ by (194). For the other direction, let $\mathbf{v} \in$ $H^{1}\left(\Omega, \mathbb{R}^{3}\right)$ be arbitrary, and let $f=\mathbf{n} \cdot \nabla \times\left.\mathbf{v}\right|_{\Gamma}$. By (209) or otherwise, $f \in$ $H^{-1 / 2}(\Gamma)$ and $\int_{\Gamma_{i}} f=0$ for all $i$, hence $f \in \mathcal{S}_{\Gamma}$. This shows that for $N=3$,

$$
\mathcal{S}_{\Gamma}=\mathbf{n} \cdot \nabla \times H^{1}\left(\Omega, \mathbb{R}^{3}\right) .
$$


A related point is that for $N=3$, the space of Stokes pressure gradients $\nabla \mathcal{S}_{p}$ (range of $[\Delta, \mathcal{P}]$ ) can be characterized as the space of simultaneous gradients and curls.

Theorem 9 Assume $\Omega \subset \mathbb{R}^{3}$ is a bounded, connected domain and its boundary $\Gamma$ is of class $C^{3}$. Then

$$
[\Delta, \mathcal{P}] H^{2}\left(\Omega, \mathbb{R}^{3}\right)=\nabla \mathcal{S}_{p}=\nabla H^{1}(\Omega) \cap \nabla \times H^{1}\left(\Omega, \mathbb{R}^{3}\right) .
$$

Proof: Indeed, $\nabla \mathcal{S}_{p} \subset \nabla \times H^{1}$ by (209) and Theorem 8. On the other hand, if $\mathbf{g}=\nabla \times \mathbf{v}=\nabla p$ then $\Delta p=\nabla \cdot \mathbf{g}=0$ and $\left.\mathbf{n} \cdot \nabla p\right|_{\Gamma} \in \mathcal{S}_{\Gamma}$ by (209) and Theorem 8 , so $\nabla p \in \nabla \mathcal{S}_{p}$.

Remark 4. In the book [Te1] (see Theorem 1.5) Temam establishes the orthogonal decomposition $L^{2}\left(\Omega, \mathbb{R}^{N}\right)=H \oplus H_{1} \oplus H_{2}$, which means that for any $g \in L^{2}\left(\Omega, \mathbb{R}^{N}\right)$,

$$
\mathbf{g}=\mathcal{P} \mathbf{g}+\nabla q+\nabla \Delta^{-1} \nabla \cdot \mathbf{g}
$$

where $q$ satisfies $\Delta q=0$ and $\left.\mathbf{n} \cdot \nabla q\right|_{\Gamma}=\mathbf{n} \cdot\left(\mathbf{g}-\nabla \Delta^{-1} \nabla \cdot \mathbf{g}\right)$. By contrast, we have shown

$$
\mathbf{g}=\mathcal{P} \mathbf{g}+\nabla p+\nabla \nabla \cdot \Delta^{-1} \mathbf{g}
$$

where $p$ satisfies $\Delta p=0$ and $\left.\mathbf{n} \cdot \nabla p\right|_{\Gamma}=\mathbf{n} \cdot\left(\mathbf{g}-\nabla \nabla \cdot \Delta^{-1} \mathbf{g}\right)$, i.e., $p$ is the Stokes pressure associated with $\Delta^{-1} \mathbf{g}$. Thus the map $\mathbf{g} \mapsto \nabla p-\nabla q$ is the commutator $\nabla \Delta^{-1} \nabla \cdot-\nabla \nabla \cdot \Delta^{-1}$. The decomposition (212) is orthogonal, and $q$ satisfies $\int_{\Gamma} \mathbf{n} \cdot \nabla q=0$. In our decomposition (213), the gradient terms are not orthogonal, but the Stokes pressure term enjoys the bounds stated in Theorem 1 , and if $\Gamma$ is not connected, it has the extra property that $\int_{\Gamma_{i}} \mathbf{n} \cdot \nabla p=0$ for every $i$.

\section{Equivalence with a gauge method}

In this appendix we show that the scheme (153)-(155) from Section 7 is equivalent to a first-order gauge method essentially the same as studied in [EL, WL]. A similar observation is made in [GMS] for the linear case with smooth solutions and homogeneous boundary conditions.

At the time-continuous level, the idea is to reformulate the unconstrained formulation (135)-(136) using the representation

$$
\mathbf{u}=\mathbf{a}+\nabla \Phi
$$

for an appropriate gauge $\Phi$. We require

$$
\begin{array}{rc}
\partial_{t} \mathbf{a}+\mathcal{P}(\mathbf{u} \cdot \nabla \mathbf{u}-\mathbf{f})=\nu \Delta \mathbf{a} & \text { in } \Omega, \\
\mathbf{a}+\nabla \Phi=\mathbf{g} & \text { on } \Gamma, \\
\partial_{t} \Phi+\nu p_{\mathrm{S}}+p_{g h}=\nu \Delta \Phi-\nu \bar{h} & \text { in } \Omega, \\
\mathbf{n} \cdot \nabla \Phi=\mathbf{n} \cdot \mathbf{g} & \text { on } \Gamma .
\end{array}
$$


Here $p_{g h}$ is determined via (135), $p_{\mathrm{S}}$ is the Stokes pressure satisfying $\nabla p_{\mathrm{S}}=$ $(\Delta \mathcal{P}-\mathcal{P} \Delta) \mathbf{u}$, and $\bar{h}$ is the average value of $h$. Adding the gradient of $(217)$ to (215) produces (136).

When initial data satisfy $\nabla \cdot \mathbf{a}+\Delta \Phi=h$ at $t=0,(217)$ can be replaced by

$$
\nabla \cdot \mathbf{a}+\Delta \Phi=h \quad \text { in } \Omega .
$$

This has advantages for computational purposes and is used in the discretization below. If we define $\psi$ by solving

$$
\Delta \psi=h \quad \text { in } \Omega, \quad \mathbf{n} \cdot \nabla \psi=\mathbf{n} \cdot \mathbf{g} \quad \text { on } \Gamma,
$$

then $(219)$ implies $\nabla(\Phi-\psi)=(\mathcal{P}-I) \mathbf{a}$ and hence $\mathbf{u}=\mathbf{a}+\nabla \Phi=\mathcal{P} \mathbf{a}+\nabla \psi$.

Time discretization of the gauge formulation above yields a scheme that can be written in the following form, convenient for our analysis. First, determine $\psi^{n}$ for all $n \geq 0$ as the mean-zero solution of

$$
\Delta \psi^{n}=h^{n} \quad \text { in } \Omega, \quad \mathbf{n} \cdot \nabla \psi^{n}=\mathbf{n} \cdot \mathbf{g}^{n} \quad \text { on } \Gamma .
$$

Then $\left\langle\nabla \psi^{n}, \nabla \phi\right\rangle=\left\langle\mathbf{n} \cdot \mathbf{g}^{n}, \phi\right\rangle_{\Gamma}-\left\langle h^{n}, \phi\right\rangle$ for all $\phi \in H^{1}(\Omega)$. Assuming the data are as in section 7.2 we have $h^{n} \in H^{1}(\Omega), \mathbf{g}^{n} \in H^{3 / 2}(\Gamma)$ and hence $\psi^{n} \in H^{3}(\Omega)$. Using (155) one can determine that

$$
\frac{\psi^{n+1}-\psi^{n}}{\Delta t}-\nu \Delta \psi^{n+1}=-p_{g h}{ }^{n}-\nu \bar{h}^{n+1},
$$

where $\bar{h}^{n+1}$ is the average value of $h^{n+1}$.

Now we write a gauge method as follows. Given $\mathbf{a}^{n}$ at time $t_{n}=n \Delta t$ such that $\mathbf{n} \cdot \mathbf{a}^{n}=0$ on $\Gamma$, find $\mathbf{u}^{n}$ and $\phi^{n}$ so that

$$
\begin{aligned}
\mathbf{u}^{n} & =\mathcal{P} \mathbf{a}^{n}+\nu \Delta t(I-\mathcal{P}) \Delta \mathbf{a}^{n}+\nabla \psi^{n}, \\
\Delta \phi^{n} & =-\nabla \cdot \mathbf{a}^{n} \quad \text { in } \Omega, \quad \mathbf{n} \cdot \nabla \phi^{n}=0 \quad \text { on } \Gamma,
\end{aligned}
$$

then determine $\mathbf{a}^{n+1}$ by solving

$$
\begin{aligned}
& \frac{\mathbf{a}^{n+1}-\mathbf{a}^{n}}{\Delta t}+\mathcal{P}\left(\mathbf{u}^{n} \cdot \nabla \mathbf{u}^{n}-\mathbf{f}^{n}\right)=\nu \Delta \mathbf{a}^{n+1} \quad \text { in } \Omega, \\
& \mathbf{a}^{n+1}+\nabla \phi^{n}+\nabla \psi^{n+1}=\mathbf{g}^{n+1} \quad \text { on } \Gamma \text {. }
\end{aligned}
$$

Since $(224)$ means $\nabla \phi^{n}=(\mathcal{P}-I) \mathbf{a}^{n}$, from (223) and (225) we have

$$
\mathbf{u}^{n+1}=\mathbf{a}^{n+1}+\nabla \phi^{n}+\nabla \psi^{n+1}
$$

for $n \geq 0$. For computational purposes, one would update the velocity using this equation, and compute $\phi^{n}+\psi^{n+1}$ from a single boundary-value problem. We need (223) here for initialization, but note that in general this equation determines $\mathbf{a}^{n}$ from $\mathbf{u}^{n}$ as well as vice-versa - see below.

One can compare (225)-(227) with (214)-(216) taking $\Phi=\phi+\psi$. To see the connection with (217)-(218), see (221)-(222) and (232) below. 
Proposition 4 The scheme (153)-(155) is equivalent to the gauge method (223)(226).

Proof: First suppose that we have a solution of the gauge method (223)-(226), with $\mathbf{a}^{n} \in H^{2}\left(\Omega, \mathbb{R}^{N}\right)$ and (hence) $\phi^{n} \in H^{3}(\Omega)$. We need to assume in addition that $(I-\mathcal{P}) \Delta \mathbf{a}^{0} \in H^{2}\left(\Omega, \mathbb{R}^{N}\right)$, so that $\mathbf{u}^{0} \in H^{2}\left(\Omega, \mathbb{R}^{N}\right)$. Observe that

$$
\mathcal{P} \mathbf{u}^{n}=\mathcal{P} \mathbf{a}^{n} \quad \text { and } \quad \mathcal{P} \Delta \mathbf{u}^{n}=\mathcal{P} \Delta \mathbf{a}^{n},
$$

which follows since $\mathbf{u}^{n}-\mathbf{a}^{n}$ is the gradient of an $H^{3}$ function, by (227) for $n>0$. Next, we can write $(I-\mathcal{P}) \mathbf{u}^{n}=\nabla q^{n}$ where $q^{n} \in H^{3}(\Omega)$ has mean zero. Since

$$
(I-\mathcal{P}) \Delta \mathbf{a}^{n}=\Delta(I-\mathcal{P}) \mathbf{a}^{n}+(\Delta \mathcal{P}-\mathcal{P} \Delta) \mathbf{a}^{n}=\nabla\left(-\Delta \phi^{n}+p_{\mathrm{s}}^{n}\right),
$$

applying $(I-\mathcal{P})$ to $(223)$ we find $q^{n}$ is determined from $\phi^{n}$ (or vice versa) by

$$
\nu \Delta \phi^{n}-\nu p_{\mathrm{s}}^{n}=\frac{\psi^{n}-q^{n}}{\Delta t} \quad \text { in } \Omega, \quad \mathbf{n} \cdot \nabla \phi^{n}=0 \quad \text { on } \Gamma .
$$

Applying $\mathcal{P}$ and $(\mathcal{P}-I)$ respectively to $(225)$, we get

$$
\begin{aligned}
& \frac{\mathcal{P} \mathbf{u}^{n+1}-\mathcal{P} \mathbf{u}^{n}}{\Delta t}+\mathcal{P}\left(\mathbf{u}^{n} \cdot \nabla \mathbf{u}^{n}-\mathbf{f}^{n}\right)=\nu \mathcal{P} \Delta \mathbf{u}^{n+1}, \\
& \frac{\phi^{n+1}-\phi^{n}}{\Delta t}=\nu \Delta \phi^{n+1}-\nu p_{\mathrm{S}}^{n+1}
\end{aligned}
$$

Using (230) it follows $\phi^{n+1}-\phi^{n}=\psi^{n+1}-q^{n+1}$ and so

$$
\nu \Delta q^{n+1}=\nu \Delta\left(\psi^{n+1}+\phi^{n}-\phi^{n+1}\right) .
$$

By combining these equations with (222), we find

$$
\frac{q^{n+1}-q^{n}}{\Delta t}-\nu \Delta q^{n+1}=-p_{g h}{ }^{n}-\nu \bar{h}^{n+1}+\nu p_{\mathrm{S}}^{n+1}-\nu p_{\mathrm{S}}^{n} .
$$

Taking the gradient, and using $\Delta \nabla q^{n+1}+\nabla p_{\mathrm{S}}^{n+1}=(I-\mathcal{P}) \Delta \mathbf{u}^{n+1}$, we get

$$
(I-\mathcal{P})\left(\frac{\mathbf{u}^{n+1}-\mathbf{u}^{n}}{\Delta t}\right)+\nabla p_{g h}{ }^{n}+\nu \nabla p_{\mathrm{s}}^{n}=\nu(I-\mathcal{P}) \Delta \mathbf{u}^{n+1} .
$$

Together with (231) and (227) this yields the scheme (153)-(155).

Next we argue in the other direction. Suppose that we have a solution of (153)-(155) with $\mathbf{u}^{n} \in H^{2}\left(\Omega, \mathbb{R}^{N}\right)$ for $n \geq 0$. Then we can determine $\mathbf{a}^{n}$ from $\mathbf{u}^{n}$ so that (223) holds by setting

$$
\mathbf{a}^{n}=\mathcal{P} \mathbf{u}^{n}-\nabla \phi^{n},
$$

where $\phi^{n}$ is obtained by solving (230) with $\nabla p_{\mathrm{s}}^{n}=(\Delta \mathcal{P}-\mathcal{P} \Delta) \mathbf{u}^{n}$. Then $\nabla \phi^{n}=$ $(\mathcal{P}-I) \mathbf{a}^{n}$ and (224) holds, and since $\phi^{n} \in H^{3}(\Omega)$ we get also (228) and (229). Applying $\mathcal{P}$ to $(153)$ yields $(231)$. Applying $(I-\mathcal{P})$ to $(153)$ we obtain $(235)$ 
and hence (234). Using this together with (222) and (230) leads to (233). Since all quantities in (232) have mean zero and $\mathbf{n} \cdot \nabla q^{n+1}=\mathbf{n} \cdot \mathbf{u}^{n+1}=\mathbf{n} \cdot \mathbf{g}^{n+1}=$ $\mathbf{n} \cdot \nabla \psi^{n+1}$ on $\Gamma$, we infer that (232) holds. This ensures

$$
(I-\mathcal{P})\left(\frac{\mathbf{a}^{n+1}-\mathbf{a}^{n}}{\Delta t}\right)=\nu \nabla\left(-\Delta \phi^{n+1}+p_{\mathrm{S}}^{n+1}\right)=\nu(I-\mathcal{P}) \Delta \mathbf{a}^{n+1} .
$$

Combining this with (231) and (228) gives (225), and (227) and (226) follow. This concludes the proof.

\section{References}

[Am1] H. Amann, On the strong solvability of the Navier-Stokes equations, J. Math. Fluid Mech. 2 (2000) 16-98.

[Am2] H. Amann, Nonhomogeneous Navier-Stokes equations with integrable low-regularity data, in Nonlinear problems in mathematical physics and related topics II, Int. Math. Ser., Kluwer/Plenum, New York (2002), 128.

[Au] T. Aubin, Nonlinear analysis on manifolds, Monge-Ampère equations. Springer-Verlag, New York, 1982.

[Ba] J. M. Ball, Strongly continuous semigroups, weak solutions, and the variation of constants formula. Proc. Amer. Math. Soc. 63 (1977) 370-373.

$[\mathrm{Bm}]$ J. W. Baumgarte, A new method of stabilization for holonomic constraints, J. Appl. Mech. Transactions ASME 50 (1983)869-870.

[BBGS] T. Barth, P. Bochev, M. Gunzburger and J. Shadid, A taxonomy of consistently stabilized finite element methods for the Stokes problem, SIAM J. Sci. Comput. 25 (2004) 1585-1607.

$[\mathrm{BCM}]$ D. L. Brown, R. Cortez and M. L. Minion, Accurate projection methods for the incompressible Navier-Stokes equations, J. Comp. Phys. 168 (2001) 464-499.

[Ch1] A. J. Chorin, Numerical solution of the Navier-Stokes equations, Math. Comp., 22 (1968) 745-762

[Ch2] A. J. Chorin, On the convergence of discrete approximations to the Navier-Stokes equations, Math. Comp. 23 (1969) 341-353.

[EL] W. E and J.-G. Liu, Gauge method for viscous incompressible flows, Commun. Math. Sci. 1(2) (2003) 317-332.

[Ev] L. C. Evans, Partial Differential Equations, Amer. Math. Soc., Providence, 1998.

[FB] M. Fortin and F. Brezzi, Mixed and Hybrid Finite Elements Methods, Springer-Verlag, New York, 1991.

[Fr] A. Friedman, Partial Differential Equations, Holt, Rinehart and Winston, New York, 1969. 
$[\mathrm{Fu}]$ A. Fursikov, M. Gunzburger and L. Hou, Trace theorems for threedimensional, time-dependent solenoidal vector fields and their applications, Trans. Amer. Math. Soc. 354 (2002), 1079-1116.

[GR] V. Girault and P-A. Raviart, Finite Element Methods for Navier-Stokes Equations: Theory and Algorithms. Springer-Verlag, Berlin, 1986.

[Gr] G. Grubb, Nonhomogeneous Dirichlet Navier-Stokes problems in low regularity $L_{p}$ Sobolev spaces, J. Math. Fluid Mech. 3 (2001), 57-81.

[GS1] G. Grubb and V. A. Solonnikov, Reduction of the basic initial-boundary value problems for the Navier-Stokes equations to initial-boundary value problems for nonlinear parabolic systems of pseudodifferential equations, J. Soviet Math. 56 (1991) 2300-2308.

[GS2] G. Grubb and V. A. Solonnikov, Boundary value problems for the nonstationary Navier-Stokes equations treated by pseudodifferential methods, Math. Scand. 69 (1991) 217-290.

[GT] D. Gilbarg and N.S. Trudinger, Elliptic Partial Differential Equations of Second Order, 3rd ed, Springer-Verlag, Berlin, 1998.

[GMS] J. L. Guermond, P. Minev and J. Shen, An overview of projection methods for incompressible flows, Comput. Methods Appl. Mech. Eng., to appear.

[GuS] J. L. Guermond and J. Shen, A new class of truly consistent splitting schemes for incompressible flows, J. Comp. Phys. 192 (2003) 262-276.

[HP] W. D. Henshaw and N. A. Petersson, A split-step scheme for the incompressible Navier-Stokes equations, in Numerical simulations of incompressible flows (Half Moon Bay, CA, 2001), 108-125, World Sci. Publishing, River Edge, NJ, 2003.

[KM] J. Kim and P. Moin, Application of a fractional-step method to incompressible Navier-Stokes equations. J. Comp. Phys. 59 (1985) 308-323.

[JL] H. Johnston and J.-G. Liu, Accurate, stable and efficient Navier-Stokes solvers based on explicit treatment of the pressure term, J. Comp. Phys. 199 (1) (2004) 221-259.

[La] O. A. Ladyzhenskaya, The Mathematical Theory of Viscous Incompressible Flow, Gordon and Breach, New York, 1969.

[Li] P. Lin, A sequential regularization method for time-dependent incompressible Navier-Stokes equations, SIAM J. Num. Anal. 34 (1997) 10511071.

[LM] J. L. Lions and E. Magenes, Non-homogeneous Boundary Value Problems and Applications, vol. I and II. Springer-Verlag, Berlin, 1972

[OID] S. A. Orszag, M. Israeli and M. Deville, Boundary conditions for incompressible flows. J. Sci. Comput. 1, (1986) 75-111.

[Pa] A. Pazy, Semigroups of Linear Operators and Applications to Partial Differential Equations, Springer-Verlag, New York, 1983. 
[Pe] N. A. Petersson, Stability of pressure boundary conditions for Stokes and Navier-Stokes equations, J. Comp. Phys. 172 (2001) 40-70.

[RR] M. Renardy and R. C. Rogers. An Introduction to Partial Differential Equations. Springer-Verlag, New York, 1993.

[Sa] M. S. Sikiru Adigun Sanni, A coupled system of the Reynolds', $k-\varepsilon$ and scalar concentration equations, Dissertation, Universität Leipzig, 2004.

[Soh] H. Sohr, The Navier-Stokes Equations, an Elementary Functional Analytic Approach. Birkhäuser Verlag, Basel, 2001.

[Sol] V. A. Solonnikov, Estimates of the solutions of a nonstationary linearized system of Navier-Stokes equations. Amer. Math. Soc. Transl. 75, (1968) $1-116$.

[Tar] L. Tartar, Topics in Nonlinear Analysis. Publications mathématiques d'Orsay 78.13. Université de Paris Sud, France, 1978.

[Tay] M. Taylor, Partial Differential Equations: Basic Theory. SpringerVerlag, New York, 1996.

[Te1] R. Temam, Navier-Stokes Equations: Theory and Numerical Analysis. AMS Chelsea, Providence, 2001.

[Te2] R. Temam, Sur l'approximation de la solution des equations de NavierStokes par la méthode des fractionnarires II, Arch. Rational Mech. Anal., 33, (1969) 377-385.

[Ti] L. J. P. Timmermans, P. D. Minev, F. N. Van De Vosse, An approximate projection scheme for incompressible flow using spectral elements, Int. J. Numer. Methods Fluids 22 (1996) 673-688.

[WL] C. Wang and J.-G. Liu, Convergence of gauge method for incompressible flow, Math. Comp. 69 (2000) 1385-1407. 